\title{
COUNTERFACTUAL EXPLANATIONS WITHOUT OPENING THE BLACK BOX: AUTOMATED DECISIONS AND THE GDPR
}

\author{
Sandra Wachter ${ }^{1,2^{*}}$, Brent Mittelstadt ${ }^{2,1,3}$, Chris Russell ${ }^{2,4}$ \\ ${ }^{1}$ Oxford Internet Institute, University of Oxford, 1 St. Giles, Oxford, \\ OX1 3JS, UK \\ ${ }^{2}$ The Alan Turing Institute, British Library, 96 Euston Road, London, \\ NW1 2DB, UK \\ ${ }^{3}$ Department of Science and Technology Studies, University College \\ London, 22 Gordon Square, London, WC1E 6BT, UK \\ ${ }^{4}$ Department of Electrical and Electronic Engineering, University of \\ Surrey, Guildford, GU2 7HX, UK \\ *E-mail: sandra.wachter@oii.ox.ac.uk. Corresponding author.
}

\section{ABSTRACT}

There has been much discussion of the "right to explanation" in the EU General Data Protection Regulation, and its existence, merits, and disadvantages. Implementing a right to explanation that opens the 'black box' of algorithmic decision-making faces major legal and technical barriers. Explaining the functionality of complex algorithmic decisionmaking systems and their rationale in specific cases is a technically challenging problem. Some explanations may offer little meaningful information to data subjects, raising questions around their value. Data controllers have an interest to not disclose information about their algorithms that contains trade secrets, violates the rights and freedoms of others (e.g. privacy), or allows data subjects to game or manipulate decision-making.

Explanations of automated decisions need not hinge on the general public understanding how algorithmic systems function. Even though interpretability is of great importance and should be pursued, explanations can, in principle, be offered without opening the black box. Looking at explanations as a means to help a data subject act rather than merely understand, one can gauge the scope and content of explanations according to the specific goal or action they are intended to support.

From the perspective of individuals affected by automated decision-making, we propose three aims for explanations: (1) to inform 
and help the individual understand why a particular decision was reached, (2) to provide grounds to contest the decision if the outcome is undesired, and (3) to understand what could be changed to receive a desired result in the future, based on the current decision-making model. We assess how each of these goals finds support in the GDPR, and the extent to which they hinge on opening the 'black box'. We suggest data controllers should offer a particular type of explanation, 'unconditional counterfactual explanations', to support these three aims. These counterfactual explanations describe the smallest change to the world that would obtain a desirable outcome, or to arrive at a "close possible world." As multiple variables or sets of variables can lead to one or more desirable outcomes, multiple counterfactual explanations can be provided, corresponding to different choices of nearby possible worlds for which the counterfactual holds. Counterfactuals describe a dependency on the external facts that lead to that decision without the need to convey the internal state or logic of an algorithm. As a result, counterfactuals serve as a minimal solution that bypasses the current technical limitations of interpretability, while striking a balance between transparency and the rights and freedoms of others (e.g. privacy, trade secrets).

\section{KEYWORDS}

accountability; algorithms; artificial intelligence; auditing; automated decision-making; counterfactuals; data ethics; data protection; explainability; GDPR; interpretability; machine learning; right to explanation; transparency

\section{FUNDING}

This work was supported by The Alan Turing Institute under the EPSRC grant EP/N510129/1. 


\section{TABLE OF CONTENTS}

INTRODUCTION 4

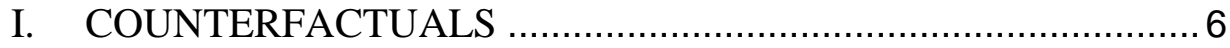

A. HISTORIC CONTEXT AND THE PROBLEM OF KNOWLEDGE7

B. EXPLANATIONS IN A.I. AND MACHINE LEARNING 10

C. ADVERSARIAL PERTURBATIONS AND COUNTERFACTUAL

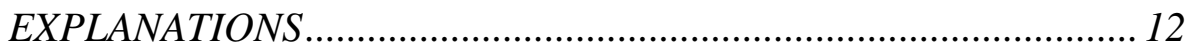

D. CAUSALITY AND FAIRNESS .................................................. 14

II. GENERATING COUNTERFACTUALS ................................. 15

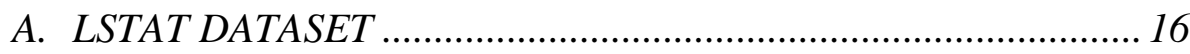

B. PIMA INDIANS DIABETES DATABASE ................................... 18

C. CAUSAL ASSUMPTIONS AND COUNTERFACTUAL

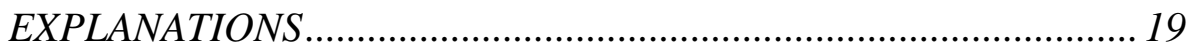

III. ADVANTAGES OF COUNTERFACTUAL EXPLANATIONS . 20

IV. COUNTERFACTUAL EXPLANATIONS AND THE GDPR .....21

A. EXPLANATIONS TO UNDERSTAND DECISIONS .................... 23

1. Broader Possibilities with the Right of Access ....................... 29

2. Understanding through Counterfactuals.................................. 31

B. EXPLANATIONS TO CONTEST DECISIONS............................... 32

1. Contesting through Counterfactuals .......................................... 37

C. EXPLANATIONS TO ALTER FUTURE DECISIONS.................. 38

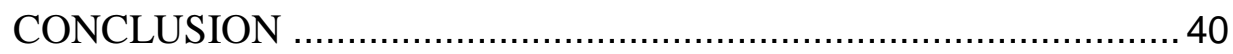

APPENDIX 1: SIMPLE LOCAL MODELS AS EXPLANATIONS ... 44 APPENDIX 2: EXAMPLE TRANSPARENCY INFOGRAPHIC ....... 47 


\section{INTRODUCTION}

There has been much discussion of the existence of a "right to explanation" in the EU General Data Protection Regulation (GDPR), and its merits and disadvantages. ${ }^{1}$ Attempts to implement a right to explanation that opens the 'black box' to provide insight into the internal decision-making process of algorithms face four major legal and technical barriers. First, a legally binding right to explanation does not exist in the GDPR. Second, even if legally binding, the right would only apply in limited cases (when a negative decision was solely automated and had legal or other similar significant effects). ${ }^{2}$ Third, explaining the functionality of complex algorithmic decision-making systems and their rationale in specific cases is a technically challenging problem. Explanations may likewise offer little meaningful information to data subjects, raising questions about their value. Finally, data controllers have an interest in not sharing details of their algorithms to avoid disclosing trade secrets, violating the rights and freedoms of others (e.g. privacy), and allowing data subjects to game or manipulate the decisionmaking system. ${ }^{3}$

Despite these difficulties, the social and ethical value (and perhaps responsibility) of offering explanations to affected data subjects remains unaffected. One significant point has been neglected in this discussion. An explanation of automated decisions, both as envisioned by the GDPR and in general, does not necessarily hinge on the general public understanding how algorithmic systems function. Even though such interpretability is of great importance and should be pursued, explanations can, in principle, be offered without opening the 'black

\footnotetext{
${ }^{1}$ Sandra Wachter, Brent Mittelstadt \& Luciano Floridi, Why a Right to Explanation of Automated Decision-Making Does Not Exist in the General Data Protection Regulation, INT. DATA PRIV. LAW (2017), https://papers.ssrn.com/sol3/papers.cfm?abstract_id=2903469; ISAK MENDOZA \& LEE A. Bygrave, The Right Not to Be Subject to Automated Decisions Based on PROFILING (2017), https://papers.ssrn.com/abstract=2964855 (last visited May 18, 2017); LiLian EdWARds \& Michael Veale, Slave to the Algorithm? Why a “Right TO AN EXPLANATION" IS PROBABLY Not THE REMEDY YOU ARE LOOKING For (2017), https://papers.ssrn.com/abstract=2972855 (last visited Aug 12, 2017); Gianclaudio Malgieri \& Giovanni Comandé, Why a Right to Legibility of Automated Decision-Making Exists in the General Data Protection Regulation, INT. DATA PRIV. LAW (2017), https://academic.oup.com/idpl/advancearticle/doi/10.1093/idpl/ipx019/4626991 (last visited Dec 18, 2017).

${ }^{2}$ Wachter, Mittelstadt, and Floridi, supra note 1.

${ }^{3}$ Jenna Burrell, How the Machine "Thinks:" Understanding Opacity in Machine Learning Algorithms, BIG DATA SOC. (2016).
} 
box'. Looking at explanations as a means to help a data subject act rather than merely understand, one could gauge the scope and content of explanations according to the specific goal or action they are intended to support.

Explanations can serve many purposes. To investigate the potential scope of explanations it seems reasonable to start from the perspective of the data subject. We propose three aims for explanations to assist data subjects: (1) to inform and help the subject understand why a particular decision was reached, (2) to provide grounds to contest adverse decisions, and (3) to understand what could be changed to receive a desired result in the future, based on the current decisionmaking model. As we show, the GDPR offers little support to achieve any of these aims. However, none hinge on explaining the internal logic of automated decision-making systems.

Building trust is essential to increase societal acceptance of algorithmic decision-making. As a solution to close current gaps in transparency and accountability that undermine trust between data controllers and data subjects, we propose to move beyond the limitations of the GDPR. We argue that counterfactuals should be used as a means to provide explanations for individual decisions.

Unconditional counterfactual explanations should be given for positive and negative automated decisions, and regardless of whether the decisions are solely (as opposed to predominantly) automated, or produce legal or significant effects. This approach provides data subjects with meaningful explanations to understand a given decision, grounds to contest it and advice on how the data subject can change their behaviour or situation to possibly receive a desired decision (e.g. loan approval) in the future, without facing the severely limited applicability imposed by the GDPR's definition of automated individual decision-making.

In this paper, we present the concept of unconditional counterfactual explanations as a novel type of explanation of automated decisions that overcomes many challenges facing current work on algorithmic interpretability and accountability. We situate counterfactuals in the philosophical history of knowledge, as well as historical and modern research on interpretability and fairness in machine learning. Based on the potential advantages offered to data subjects by counterfactual explanations, we then assess their alignment with the GDPR's numerous provisions concerning automated decisionmaking. Specifically, we examine whether the GDPR offers support for explanations that aim to help data subjects understand the scope of 
automated decision-making and rationale of specific decisions; explanations to contest decisions; and explanations that offer guidance on how data subjects can change their behaviour to receive a desired result. We conclude that unconditional counterfactual explanations can bridge the gap between the interests of data subjects and data controllers, which otherwise act as a barrier to a legally binding right to explanation.

\section{COUNTERFACTUALS}

Counterfactual explanations take a similar form to the statement:

"You were denied a loan because your annual income was $£ 30,000$. If your income had been $£ 45,000$ you would have been offered a loan."

Here the statement of decision is followed by a counterfactual, or statement of how the world would have to be different for a desirable outcome to occur. Multiple counterfactuals are possible, as multiple desirable outcomes can exist, and there may be several ways to achieve any of these outcomes. The concept of "closest possible world," or the smallest change to the world that can be made to obtain a desirable outcome, is key throughout the discussion of counterfactuals. In many situations, providing several explanations covering a range of diverse counterfactuals, corresponding to relevant or informative "close possible worlds" rather than "the closest possible world" may be more helpful. Knowing the smallest possible change to a variable or set of variables to arrive at a different outcome may not always be the most helpful type of counterfactual. Rather, relevance will depend also upon other casespecific factors, such as the mutability of a variable or real world probability of a change (see Section \#).

In the existing literature, "explanation" typically refers to an attempt to convey the internal state or logic of an algorithm that leads to a decision. ${ }^{4}$ In contrast, counterfactuals describe a dependency on the external facts that led to that decision. This is a crucial distinction. In modern machine learning, the internal state of the algorithm can consist of millions of variables intricately connected in a large web of dependent behaviours. Conveying this state to a layperson in a way that allows them to reason about the behaviour of an algorithm is extremely challenging.

${ }^{4} I d$. 
The machine learning and legal communities have both taken relatively restricted views on what passes for an explanation. The machine learning community has been primarily concerned with debugging and conveying approximations of algorithms that programmers or researchers could use to understand which features are important; while law and ethics scholars have been more concerned with understanding the internal logic of decisions as a means to assess their lawfulness (e.g. prevent discriminatory outcomes), contest them, increase accountability generally, and clarify liability.

As such, our proposal for counterfactuals as explanations lies outside of the taxonomies of explanations proposed previously in machine learning, legal and ethical literature. In contrast, analytic philosophy has taken a much broader view of knowledge and how counterfactuals can be used as justifications of beliefs (or in our case, decisions).

\section{A. HISTORIC CONTEXT AND THE PROBLEM OF KNOWLEDGE}

Analytic Philosophy has a long history of analysing the necessary conditions for propositional knowledge. Expressions of the type ' $S$ knows that $p$ ' constitute knowledge, "where $S$ refers to the knowing subject, and $p$ to the proposition that is known." Traditional approaches, which conceive of knowledge as 'justified true belief', conceive of three necessary conditions: truth, belief, and justification. According to this approach, in order to know something, it is not enough to simply believe that something is true, but that you must also have a good reason for believing it. Our interest in this literature comes from the observation that such justifications of belief can serve as explanations, as fundamentally they are a reason that a belief is held, and therefore serve as an answer to the question "Why do you believe $X$ ?". Therefore, understanding the different forms these justifications can take opens the door to a broader class of explanations than previously encountered in interpretability research.

Although influential, 'justified true belief' has faced much criticism and inspired substantial analysis of modifications to the tripartite, and proposals of additional necessary conditions for a 
proposition to constitute knowledge. ${ }^{5}$ Modal conditions, including safety ${ }^{6}$ and sensitivity, ${ }^{7}$ have been proposed as necessary additions to the tripartite built on counterfactual relations. ${ }^{8}$

Following Sosa ${ }^{9}$, Ichikawa and Steup ${ }^{10}$ define sensitivity as:

If $p$ were false, $S$ would not believe that $p$.

Here, the statement "If $p$ were false" is a counterfactual defining a "possible world" close to the world in which $p$ is true. ${ }^{11}$ The sensitivity condition suggests that "in the nearest possible worlds in which not- $p$, the subject does not believe that $p . " 12$ Our notion of counterfactual explanations hinges upon the related concept:

If $q$ were false, $S$ would not believe $p$.

We claim that in this case $q$ serves as an explanation of $S$ 's belief in $p$, inasmuch as $S$ only holds belief $p$ while $q$ is true, and that changing $q$ would also cause $S$ 's belief to change. A key point, worth emphasising, is that such statements only describe $S$ 's beliefs, which need not reflect reality. ${ }^{13}$ As such, these statements can be made without knowledge of any causal relationship between $q$ and $p$.

We define Counterfactual Explanations as statements taking the form:

Score $p$ was returned because variables $V$ had values $\left(v_{1}, v_{2}, \ldots\right)$ associated with them. If $V$ instead had values $\left(v_{1}{ }^{\prime}, v_{2}{ }^{\prime}, \ldots\right)$ score $p^{\prime}$ would have been returned.

\footnotetext{
${ }^{5}$ Jonathan Ichikawa \& Matthias Steup, The Analysis of Knowledge, in STANFORD ENCYCLOPEDIA OF PHILOSOPHY (Fall 2017 ed. 2017), https://plato.stanford.edu/archives/fall2017/entries/knowledge-analysis/.

${ }^{6}$ Ernest Sosa, How to Defeat Opposition to Moore, 13 PHILOS. PERSPECT. 141-153 (1999).

${ }^{7}$ Jonathan Ichikawa, Quantifiers, knowledge, and counterfactuals, 82 PHILOS. PHENOMENOL. RES. 287-313 (2011); RoBERT NOZICK, PHILOSOPHICAL EXPLANATIONS (1981).

${ }^{8}$ For an excellent review of these concepts and their criticisms see section 5 of Ichikawa and Steup, supra note 5..

${ }^{9}$ Sosa, supra note 6.

${ }^{10}$ Ichikawa and Steup, supra note 5.

${ }^{11}$ DAVID LEWIS, COUNTERFACTUALS (1973).

${ }^{12}$ Ichikawa and Steup, supra note 5.

${ }^{13}$ For example, $S$ could believe that a person is inherently more trustworthy $(p)$ because they are a Capricorn $(q)$.
} 
While many such explanations are possible, an ideal counterfactual explanation would alter values as little as possible and represent a closest world under which score $p$ ' is returned instead of $p$. The notion of a "closest possible world" is thus implicit in our definition.

Our version of counterfactuals perhaps most resembles a structural equations approach in execution by identifying alterations to variables more similar to Pearl's "mini-surgeries"14 than Lewis" "miracles." "In any case, our approach does not rely on knowledge of the causal structure of the world (see section 'Causality and Fairness'), or suggest which (context-dependent) metric of distance between worlds is preferable to establish causality. ${ }^{16}$ In many situations, it will be more informative to provide a diverse set of counterfactual explanations, corresponding to different choices of nearby possible worlds for which the counterfactual holds (or a preferred outcome is delivered), rather than a theoretically ideal counterfactual describing the "closest possible world" according to a preferred distance metric. ${ }^{17}$ Many considerations will be relevant to the choice of distance metric and a 'sufficient' and 'relevant' set of counterfactual explanations, many of which will be dependent on the facts of a specific case (e.g. capabilities of the individual concerned, sensitivity and mutability of the variables involved in a decision, ethical or legal requirements for disclosure). ${ }^{18}$

Similarly, counterfactuals that describe changes to multiple variables within the model can be provided. These would represent possible futures brought about by changes to the individual's circumstances. As an example, the impact of changes in income could be calculated in combination with changes to career, thereby ensuring the counterfactual represents a realistic possible world.

\footnotetext{
${ }^{14}$ JUdEA PEARL, CAUSATION (2000).

${ }^{15}$ LEWIS, supra note 11.

${ }^{16}$ Boris Kment, Counterfactuals and explanation, 115 MIND 261-310 (2006).

${ }^{17}$ The merits of different metrics of distance between possible worlds have long been debated in philosophy without the emergence of consensus. Meaningfully addressing this debate goes beyond the scope of this paper which proposes a method for counterfactual explanations, but will be explored in future work. For further discussion of distance metrics and counterfactuals, see: LEWIS, supra note 11; Ernest W. Adams, On the rightness of certain counterfactuals, 74 PAC. PHILOS. Q. 1-10 (1993); Kment, supra note 16.

${ }^{18}$ A discussion of appropriate metrics for making these choices goes beyond the scope of this paper, but will be addressed in future work. With that said, relevant philosophical discussion can be found on determining relevance of possible causal or contrastive explanations, counterfactuals, and distance metrics. See for example: Peter Lipton, Contrastive explanation, 27 R. InST. PHILOS. SUPPL. 247-266 (1990); Adams, supra note 17.
} 


\section{B. EXPLANATIONS IN A.I. AND MACHINE LEARNING}

Much of the early work in A.I. on explaining the decisions made by expert or rule-based systems focused on classes of explanation closely related to counterfactuals. For example, Gregor and Benbasat ${ }^{19}$ offer the following example of what they call a type 1 explanation:

Q: Why is a tax cut appropriate?

A: Because a tax cuts preconditions are high inflation and trade deficits, and current conditions include these factors.

While Buchanan and Shortliffe ${ }^{20}$ offer a similar example:

\section{RULE009 IF:}

1) The gram stain of the organism is gramneg, and

2) The morphology of the organism is coccus

THEN: There is strongly suggestive evidence (.8) that the Identity of the organism is Neisseria

As is typical in early A.I. questions we now recognise as hard such as "How do we decide inflation is high?", or "Why are these the preconditions of a tax cut?" are assumed to have been addressed by humans. As such, the explanations do not provide insight into what people in machine learning think of as the internal logic of black box classifiers. In fact, the first example can be rewritten as two diverse counterfactual statements:

"If inflation was lower, a tax cut would not be recommended."

"If there was no trade deficit, a tax cut would not be recommended."

While the second example is closely related to the counterfactual: ${ }^{21}$

"If the gram stain was negative or the morphology was not coccus, the algorithm would not be confident that the organism is Neisseria."

\footnotetext{
${ }^{19}$ Shirley Gregor \& Izak Benbasat, Explanations from intelligent systems: Theoretical foundations and implications for practice, MIS Q. 497-530 (1999).

${ }^{20}$ B. G. BUCHANAN \& E. D. SHORTLIFFE, RULE-BASED EXPERT SYSTEMS: THE MYCIN EXPERIMENTS OF THE STANFORD HEURISTIC PROGRAMMING PROJECT (1984).

${ }^{21}$ However, they are not logically equivalent. The example from MYCIN differs in that it is still possible that some samples that are either gram positive or have a different morphology could still be classified as Neisseria.
} 
The most important difference between these approaches and counterfactuals is that counterfactuals continue functioning in an end-toend integrated approach. If the gram stain and morphology in the MYCIN example were also determined by the algorithm, counterfactuals would automatically return a close sample with a different classification, while these early methods could not be applied to such involved scenarios.

As focus has switched from A.I. and logic based systems, and towards machine learning tasks such as image recognition, the notion of an explanation has come to refer to providing insight into the internal state of an algorithm, or to human understandable approximations of the algorithm. As such, the most related machine learning work to these, and to ours, is Martens and Provost. ${ }^{22}$ Uniquely, of other works in machine learning, it shares our interest in making interventions to alter the outcome of classifier responses. However, the work is firmly linked to the problem of document classification, and the only interventions it proposes involve the removal of words from documents, to stop websites being classified as "adult." The heuristic proposed cannot be easily generalised to either continuous variables, ${ }^{23}$ or even the addition of words to documents.

The majority of works in machine learning on explanations and interpreting models concern themselves with generating simple models as local approximations of decisions. ${ }^{24}$ Generally, the idea is to create a simple human-understandable approximation of a decision-making algorithm that accurately models the decision given the current inputs, but may be arbitrarily bad for different inputs. However, there are numerous difficulties with treating these approaches as explanations suitable for a lay data subject.

In general, it is unclear if these models are interpretable by nonexperts. Moreover, the utility of such approaches, outside of model debugging by expert programmers, is unclear. As we show in the

\footnotetext{
${ }^{22}$ David Martens \& Foster Provost, Explaining data-driven document classifications, (2013).

23 'Continuous variables' refers to measurements such as height or weight.

${ }^{24}$ Marco Tulio Ribeiro, Sameer Singh \& Carlos Guestrin, Why should i trust you?: Explaining the predictions of any classifier, in PROCEEDINGS OF THE 22ND ACM SIGKDD INTERNATIONAL CONFERENCE ON KNOWLEDGE DISCOVERY AND DATA MINING 1135-1144 (2016); Ramprasaath R. Selvaraju et al., Grad-CAM: Why did you say that?, ARXIV PREPR. ARXIV161107450 (2016); Karen Simonyan, Andrea Vedaldi \& Andrew Zisserman, Deep inside convolutional networks: Visualising image classification models and saliency maps, ARXIV PREPR. ARXIV13126034 (2013).
} 
supplementary materials, even in simple scenarios, these local models can produce widely varying estimates of the importance of variables, which, even in the single variable case, make it extremely difficult to reason about how a function varies as the inputs change.

These approaches make a three-way trade-off between the quality of the approximation vs. the ease of understanding the function and the size of the domain for which the approximation is valid. ${ }^{25} \mathrm{We}$ are unaware of any work on conveying the various limitations and unreliabilities of these approaches to a lay audience in such a way that they can make use of such explanations.

In contrast, counterfactual explanations are intentionally restricted. They are crafted in such a way as to provide a minimal amount of information capable of altering a decision; and they do not require the data subject to understand any of the internal logic of a model in order to make use of it. The downside to this is that individual counterfactuals may be overly restrictive, and a single counterfactual may show how a decision is based on data that is both correct, and cannot be altered by the data subject before future decisions, even if other data exists that could be amended for a favourable outcome. This could be resolved by offering multiple diverse counterfactual explanations to the data subject.

\section{ADVERSARIAL PERTURBATIONS AND COUNTERFACTUAL EXPLANATIONS}

The techniques used to generate counterfactual explanations on deep networks such as resnet are already widely studied in the machine learning literature under the name of 'Adversarial Perturbations'. Algorithms capable of computing counterfactuals are used to confuse existing classifiers by generating a synthetic data point close to an existing one such that the new synthetic data point is classified differently to the original one.

One strength of counterfactuals is that they can be efficiently and effectively computed by applying standard techniques, even to cuttingedge architectures. Some of the largest and deepest neural networks are used in the field of computer vision, particularly in image labelling tasks such as ImageNet. These classifiers have been shown to be particularly

\footnotetext{
${ }^{25}$ Osbert Bastani, Carolyn Kim \& Hamsa Bastani, Interpretability via Model Extraction, in FAT-ML 2017 (2017); Himabindu Lakkaraju et al., Interpretable \& Explorable Approximations of Black Box Models, (2017).
} 
vulnerable to a type of attack referred to as "Adversarial Perturbation"26 where small changes to a given image can result in the image being assigned to an entirely different class. For example, DeepFool ${ }^{27}$ defines an adverse perturbation of an image $x$, given a classifier, as the smallest change to $x$ such that the classification changes. Essentially, this is a counterfactual by a different name. Finding a closest possible world to $x$ such that the classifier changes is, under the right choice of distance function, the same as finding the smallest change to $x$.

Importantly, none of the standard works on Adversarial Perturbations make use of appropriate distance functions, and the majority of such approaches tend to favour making small changes to many variables, instead of providing sparse human interpretable solutions that modify only a few variables. ${ }^{28}$ Despite this, efficient computation of counterfactuals and Adversarial Perturbations is made possible by virtue of state-of-the-art algorithms being differentiable; and many optimisation techniques proposed in the Adversarial Perturbation literature are directly applicable to this problem, making counterfactual generation efficient.

One of the more challenging aspects of Adversarial Perturbations is that these small perturbations of an image are barely human perceptible, but result in drastically different classifier responses. Informally, this appears to happen because the newly generated images do not lie in the "space of real-images," but slightly outside it. This phenomenon serves as an important reminder that when computing counterfactuals by searching for a close possible world, it is at least as important that the solution found comes from a "possible world," as it is that it is close to the starting example. Further research into how data from high-dimensional and highly-structured spaces, such as natural images, can be characterised is needed before counterfactuals can be reliably used as explanations in these spaces.

\footnotetext{
${ }^{26}$ Ian J. Goodfellow, Jonathon Shlens \& Christian Szegedy, Explaining and harnessing adversarial examples, ARXIV PREPR. ARXIV14126572 (2014); Seyed-Mohsen Moosavi-Dezfooli, Alhussein Fawzi \& Pascal Frossard, Deepfool: a simple and accurate method to fool deep neural networks, in PROCEEDINGS OF THE IEEE CONFERENCE ON COMPUTER VISION AND PATTERN RECOGNITION 2574-2582 (2016); Christian Szegedy et al., Intriguing properties of neural networks, ARXIV PREPR. ARXIV13126199 (2013).

${ }^{27}$ Moosavi-Dezfooli, Fawzi, and Frossard, supra note 23.

${ }^{28}$ Jiawei Su, Danilo Vasconcellos Vargas \& Sakurai Kouichi, One pixel attack for fooling deep neural networks, ARXIV171008864 Cs STAT (2017), http://arxiv.org/abs/1710.08864 (last visited Dec 18, 2017).
} 


\section{CAUSALITY AND FAIRNESS}

Several works have approached the problem of guaranteeing that algorithms are fair i.e. that they do not exhibit a bias towards particular ethnic, gender, or other protected groups, using causal reasoning ${ }^{29}$ and counterfactuals. ${ }^{30}$ Kusner et al. ${ }^{31}$ consider counterfactuals where the subject belongs to a different race or sex and require that the decision made to remain the same under this counterfactual for it to be considered fair. In contrast, we consider counterfactuals in which the decision differs from its current state.

Many works have suggested that transparency might be a useful tool for enforcing fairness. While it is unclear how counterfactuals could be used for this purpose, it is also unclear if any form of explanation of individual decisions can in fact help. Grgic-Hlaca et al. ${ }^{32}$ showed how our intuitions can be easily misled, by understandable models, and that predominantly using features people believed to be fair, slightly increased the racism exhibited by algorithms, while decreasing accuracy. In general, the best tools for uncovering systematic biases are likely to be based upon large-scale statistical analysis and not upon explanations of individual decisions.

With that said, counterfactuals can provide evidence that an algorithmic decision is affected by a protected variable (e.g. race), which may be discriminatory. ${ }^{33}$ For the types of distance function we consider in the next section, if the counterfactuals found change someone's race, then the treatment of that individual is dependent on race. The converse statement is, however, not true. Counterfactuals which do not modify a protected attribute cannot be used as evidence that the attribute was irrelevant to the decision. This is because counterfactuals describe only a dependency between a decision and specific external facts, not all external facts.

\footnotetext{
${ }^{29}$ Niki Kilbertus et al., Avoiding Discrimination through Causal Reasoning, ARXIV PREPR. ARXIV170602744 (2017).

${ }^{30}$ Matt J. Kusner et al., Counterfactual Fairness, (2017).

${ }^{31}$ Id.

${ }^{32}$ Nina Grgic-Hlaca et al., The case for process fairness in learning: Feature selection for fair decision making, in NIPS SYMPOSIUM ON MACHINE LEARNING AND THE LAW (2016).

${ }^{33}$ Establishing the influence of a protected variable on a decision does not, by itself, prove that illegal discrimination has occurred. Mitigating factors may exist which justify the usage of a protected attribute. See for example discussion of disparate treatment in American anti-discrimination law; Solon Barocas \& Andrew D. Selbst, Big data's disparate impact, 104 CALIF. LAW REV. (2016).
} 


\section{GENERATING COUNTERFACTUALS}

In the following section, we give examples of how meaningful counterfactuals can be easily computed. Many of the standard classifiers of machine learning (including Neural Networks, Support Vector Machines and Regressors) are trained by finding the optimal set of weights $w$ that minimises an objective over a set of training data.

$$
\arg \min _{w} \sum_{i} \ell\left(f_{w}\left(x_{i}\right), y_{i}\right)+\rho(w)
$$

Where $y_{i}$ is the label for data point $x_{i}$ and $\rho(\cdot)$ is a regularizer over the weights. Replacing the label $y$ by the target $y^{\prime}$ decision we can find a new counterfactual $x^{\prime}$ that is as close to the original as possible by holding $w$ fixed and minimizing the related objective.

$$
\arg \min _{x^{\prime}} \max _{\lambda} \lambda\left(f_{w}\left(x^{\prime}\right)-y^{\prime}\right)^{2}+d\left(x_{i}, x^{\prime}\right)
$$

Where $d(\cdot, \cdot)$ is a distance function that measures how far the counterfactual $x^{\prime}$ and the original data point $x_{i}$ are from one another. In practice, maximisation over $\lambda$ is done by iteratively solving for $x^{\prime}$ and increasing $\lambda$ until a sufficiently close solution is found.

The choice of optimiser for these problems is relatively unimportant. In practice, any optimiser capable of training the classifier under Eq. (1) seems to work equally well, and we use $\mathrm{ADAM}^{34}$ for all experiments. As local minima are a concern, we initialise each run with different random values for $x^{\prime}$ and select as our counterfactual the best minimizer of Eq. (2). These different minima can be used as a diverse set of multiple counterfactuals.

Of particular importance is the choice of distance function used to decide which synthetic data point $x$ ' is closest to the original data point $x_{i}$. As a sensible first choice, which should be refined based on subject- and task-specific requirements, we suggest use of the $L_{1}$ norm, or Manhattan distance, weighted by the inverse median absolute deviation. This is written as $\mathrm{MAD}_{k}$ for the median absolute deviation of feature $k$, over the set of points $P$ :

\footnotetext{
${ }^{34}$ Diederik Kingma \& Jimmy Ba, Adam: A method for stochastic optimization, ARXIV PREPR. ARXIV14126980 (2014).
} 


$$
\operatorname{MAD}_{k}=\operatorname{median}_{j \in P}\left(\left|X_{j, k}-\operatorname{median}_{l \in P}\left(X_{l, k}\right)\right|\right)
$$

We chose $d(\cdot, \cdot)$ as:

$$
d\left(x_{i}, x^{\prime}\right)=\sum_{k \in F} \frac{\left|x_{i, k}-x_{k}^{\prime}\right|}{\operatorname{MAD}_{k}}
$$

This distance metric has several desirable properties. Firstly, it captures some of the intrinsic volatility of the space, which means that if a feature $k$ varies wildly across the dataset, a synthetic point $x$ ' may also vary this feature while remaining close to $x_{i}$, under the distance measure. The use of median absolute difference rather than the more usual standard deviation makes the measure used more robust to outliers. Of equal importance is the sparsity inducing properties of the $L_{l}$ norm. The $L_{l}$ norm is widely recognised in mathematical and machine learning circles for its tendency to induce sparse solutions in which most entries are zero when paired with an appropriate cost function. ${ }^{35}$

When computing human-understandable counterfactuals this property is highly desirable as it corresponds to counterfactuals in which only a small number of variables are changed and most remain constant, making the counterfactuals much easier to communicate and comprehend. Out of the box this measure works equally well on the examples we consider.

To demonstrate the importance of the choice of distance function, below we illustrate the impact of varying $d(\cdot, \cdot)$ on the LSAT dataset. A further challenge lies in ensuring that the synthetic counterfactual $x$ ' corresponds to a valid data point. We illustrate some of the pitfalls and remedies for dealing with discrete features when computing counterfactuals.

\section{A. LSTAT DATASET}

We first consider the generations of counterfactuals on the LSTAT ${ }^{36}$ dataset. In particular, we consider a stripped-down version used in the

\footnotetext{
${ }^{35}$ Emmanuel J. Candes, Justin K. Romberg \& Terence Tao, Stable signal recovery from incomplete and inaccurate measurements, 59 COMMUN. PURE APPL. MATH. 1207-1223 (2006).

${ }^{36}$ R. Darrell Bock \& Marcus Lieberman, Fitting a response model forn dichotomously scored items, 35 PSYCHOMETRIKA 179-197 (1970).
} 
fairness literature ${ }^{37}$ that attempts to predict students' first-year average grade on the basis of their race, grade-point average prior to law school, and law school entrance exam scores. This stripped down version of LSTAT dataset is used in the fairness literature, as classifiers trained on this data naturally exhibit bias against black people. As a result, we will find evidence of this bias in our neural network in some of the counterfactuals we generate.

We generate a three-layer fully-connected neural-network, with two hidden layers of 20 neurons each feeding into a final classifier. Even a small model like this has 941 different weights controlling its behaviour and 40 neurons that exhibit complex interdependencies, which makes conveying its internal state challenging.

Choosing $d$ as the unweighted squared Euclidean distance

$$
d\left(x_{i}, x^{\prime}\right)=\sum_{k \in F}\left(x_{i, k}-x_{k}^{\prime}\right)^{2}
$$

we consider the Counterfactual "What would have to be changed to give a predicted score of 0 ?"38 Directly solving for Eq. 2, gives the results in the central block labelled "Cf. Continuous" in Table 1.

\begin{tabular}{|c|ccc|ccc|ccc|}
\multicolumn{4}{c}{} & \multicolumn{9}{c}{ Onnormalised L2 } \\
Score & GPA & LSAT & Race & GPA & LSAT & Race & GPA & CS. Hybrid \\
\hline 0.17 & 3.1 & 39.0 & 0 & 3.0 & 39.0 & 0.3 & 1.5 & 38.4 & 0 \\
0.54 & 3.7 & 48.0 & 0 & 3.5 & 47.9 & 0.9 & -1.6 & 45.9 & 0 \\
-0.77 & 3.3 & 28.0 & 1 & 3.5 & 28.1 & -0.3 & 5.3 & 28.9 & 0 \\
-0.83 & 2.4 & 28.5 & 1 & 2.6 & 28.6 & -0.4 & 4.8 & 29.4 & 0 \\
-0.57 & 2.7 & 18.3 & 0 & 2.9 & 18.4 & -1.0 & 8.4 & 20.6 & 0
\end{tabular}

Table 1 - Unnormalied L2

Two artefacts are immediately apparent. The first is that although race should be a discrete value taking labels only 0 or 1 , corresponding to white or black respectively, a variety of meaningless values, either fractional or negative have been assigned to it. In the literature on adversarial perturbation, generally values are capped to lie within a sensible range such as $[0,1]$ to stop some of these artefacts from occurring. However, this would still allow the fractional solutions shown in the bottom two examples. Instead, we clamp the race variable forcing it to take either value 0 or 1 in two separate run-throughs, and then take as a solution the closest counterfactual found in either of the runs. These

\footnotetext{
${ }^{37}$ Chris Russell et al., When worlds collide: integrating different counterfactual assumptions in fairness, in ADVANCES IN NEURAL INFORMATION PROCESSING SYSTEMS 6396-6405 (2017).

${ }^{38}$ The scores being predicted are normalised, with 0 corresponding to the average score.
} 
results can be seen in the rightmost column "counterfactual hybrid." The algorithm now suggests always changing the race to white as part of the counterfactual. Of particular note is that the counterfactuals show that black students would get better scores.

The second artefact is that the algorithm much prefers significantly varying the GPA than the exam results, and this is down to our choice of distance function. We took as $d$, the squared Euclidean distance, and this generally prefers changes that are as small as possible and spread uniformly across all variables. However, the range of the GPA is much smaller than that of the exam scores. Adjusting for this by normalising each component by its standard deviation, i.e.

$$
d\left(x_{i}, x^{\prime}\right)=\sum_{k \in F} \frac{\left(x_{i, k}-x_{k}^{\prime}\right)^{2}}{\operatorname{std}_{j \in P}\left(x_{j, k}\right)}
$$

gives the set of counterfactuals shown in Table 2.

\begin{tabular}{|c|ccc|ccc|ccc|}
\multicolumn{4}{c}{} & \multicolumn{9}{c}{ Normalised L2 } & \multicolumn{3}{c|}{ Cf. Hybrid } \\
Score & GPA & LSAT & Race & GPA & LSAT & Race & GPA & LSAT & Race \\
\hline 0.17 & 3.1 & 39.0 & 0 & 3.0 & 37.0 & 0.2 & 3.0 & 34.0 & 0 \\
0.54 & 3.7 & 48.0 & 0 & 3.5 & 39.5 & 0.4 & 3.5 & 33.1 & 0 \\
-0.77 & 3.3 & 28.0 & 1 & 3.5 & 39.8 & 0.4 & 3.4 & 33.4 & 0 \\
-0.83 & 2.4 & 28.5 & 1 & 2.7 & 37.4 & 0.2 & 2.6 & 35.7 & 0 \\
-0.57 & 2.7 & 18.3 & 0 & 2.8 & 28.1 & -0.4 & 2.9 & 34.1 & 0
\end{tabular}

Table 2 - Normalised L2

After normalisation, the GPA remains much more consistent, and naturally remains within an expected range of values. Note that for black students race does vary under the computed counterfactual, revealing a dependence between the decision and race (which is often a legally protected attribute).

Finally, we show the use of the $L_{1}$ norm weighted by the inverse median absolute deviation (Table 3). This returns similar but sparser results to the weighted squared Euclidean distance, with the GPA not being changed under the counterfactuals.

\begin{tabular}{|c|cc} 
& \multicolumn{3}{|c}{ Original Data } \\
score & GPA & LSAT \\
0.17 & 3.1 & 39.0 \\
0.54 & 3.7 & 48.0 \\
-0.77 & 3.3 & 28.0 \\
-0.83 & 2.4 & 28.5 \\
-0.57 & 2.7 & 18.3
\end{tabular}
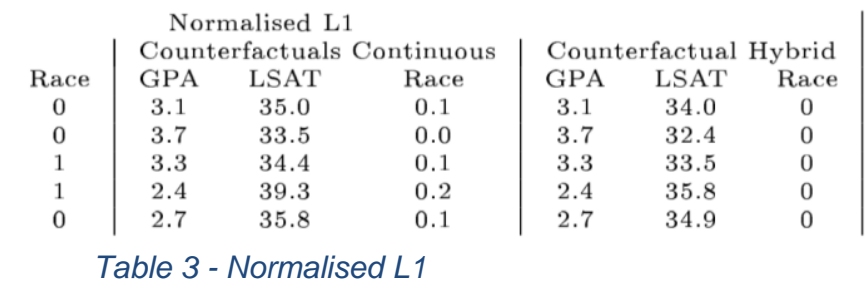

\section{B. PIMA DIABETES DATABASE}

To demonstrate Counterfactuals on a more complex problem we consider a database used to predict whether women of Pima heritage are 
at risk of diabetes ${ }^{39}$. We generate a classifier that returns a risk score between $[0,1]$ by training a similar three-layer fully-connected neuralnetwork, with two hidden layers of 20 neurons, to perform logistic regression. This classifier takes as input 10 different variables of varying predictive power, including number of pregnancies, age and BMI. Counterfactuals are generated to answer the question "What would have to be different for this individual to have a risk score of 0.5 ?" To induce sparsity in the answer, and generate counterfactuals that are easy for a human to evaluate, with only a small number of changed variables, we make use of the $L_{1}$ norm or Manhattan distance, weighted by the inverse median absolute deviation, instead of the Euclidean distance. We also cap variables to prevent them from going outside the range seen in the training data.

With this done, the counterfactuals tend to be sparse and can be rendered in human readable text form.

Person 1: If your 2-Hour serum insulin level was 154.3, you would have a score of 0.51

Person 2: If your 2-Hour serum insulin level was 169.5, you would have a score of 0.51

Person 3: If your Plasma glucose concentration was 158.3 and your 2-Hour serum insulin level was 160.5 , you would have a score of 0.51

These counterfactuals are similar to the risk factors already used by doctors to communicate e.g. "If your body mass index is greater than 40 you are morbidly obese, and at greater risk of ill-health." However, counterfactuals may make use of multiple factors and convey a personalised risk model that takes into account other attributes that may mitigate or increase risk.

\section{CAUSAL ASSUMPTIONS AND COUNTERFACTUAL EXPLANATIONS}

The reader familiar with causal modelling may have noticed that our counterfactual explanations are not making use of causal models, or equivalently that they make naive assumptions that variables are

\footnotetext{
${ }^{39}$ Jack W. Smith et al., Using the ADAP learning algorithm to forecast the onset of diabetes mellitus, in PROCEEDINGS OF THE ANNUAL SYMPOSIUM ON COMPUTER APPLICATION IN MEDICAL CARE 261 (1988).
} 
independent of one another. There are several reasons for this. One important use of counterfactual explanations is to provide the data subject with information to make a guided audit of the data, and check for relevant inaccuracies in the data. Treating such errors as independent and drawn from a robust distribution such as the Laplacian (corresponding to use of the $L_{1}$ norm in our objective), is a sensible model for these errors. More importantly, creating and interpreting accurate causal models is difficult. Requiring data controllers to build and convey to a lay audience a causal model that accurately captures the interdependencies between measurements such as the number of pregnancies, age and BMI is extremely challenging, and may be irrelevant.

Counterfactuals generated from an accurate causal model may ultimately be of use to experts e.g. to medical professionals trying to decide which intervention will move a patient out of an at risk group. However, the purpose of our paper is to illustrate how far you can go with minimal assumptions, and that such detailed causal models are unnecessary for counterfactual explanations to be of use.

\section{ADVANTAGES OF COUNTERFACTUAL EXPLANATIONS}

Counterfactual explanations differ markedly from existing proposals in the machine learning and legal communities (particularly regarding the GDPR's 'right to explanation'), ${ }^{40}$ while offering several advantages. Principally, counterfactuals bypass the substantial challenge of explaining the internal workings of complex machine learning systems. ${ }^{41}$ Even if technically feasible, such explanations may be of little practical value to data subjects. In contrast, counterfactuals provide information to the data subject that is both easily digestible and practically useful for understanding the reasons for a decision, challenging them, and altering future behaviour for a better result.

\footnotetext{
${ }^{40}$ Although a right to explanation is not itself legally binding, data subjects are entitled to receive "meaningful information about the logic involved, as well as the significance and the envisaged consequences" of automated decision-making under the GDPR's Art 13-15. Others have proposed that these provisions require the data subject to be given information about the internal logic and the rationale of specific decisions. The information sought aligns with the type of explanation pursued in the machine learning community. For an explanation of why such information is not legally required, and why Art. 13-15 do not constitute a de facto right to explanation, see Wachter, Mittelstadt, and Floridi, supra note 1.

${ }^{41}$ Burrell, supra note 3.
} 
The reduced regulatory burden of counterfactual explanations is also significant. Current state-of-the-art machine learning methods ${ }^{42}$ make decisions based upon deep networks that compose together functions more than a thousand times, and with more than ten million parameters controlling their behaviour. As the working memory of humans can contain around seven distinct items, ${ }^{43}$ it remains unclear whether human-comprehensible meaningful information' about the logic involved in a particular decision can ever exist, disregarding whether such information could be meaningfully conveyed to non-experts. ${ }^{44}$ As such regulations that require meaningful information regarding the internal logic to be conveyable to a lay audience, could prohibit the use of many standard approaches. In contrast, counterfactual explanations do not attempt to convey the logic involved and, as shown in the previous section, are simple to compute and convey.

Such expectations of providing information regarding the internal logic of algorithmic decision-making systems have surfaced recently in relation to the GDPR, and in particular the 'right to explanation'. The GDPR contains numerous provisions requiring information to be communicated to individuals about automated decision-making. Significant discussion has emerged in legal and machine learning communities regarding the specific requirements and limitations of the GDPR in this regard, and in particular how to provide information about decisions made by highly complex automated systems. As counterfactuals provide a method to explain some of the rationale of an automated decision while avoiding the major pitfalls of interpretability or opening the 'black box', they may prove a highly useful mechanism to meet the explicit requirements and background aims of the GDPR.

\section{COUNTERFACTUAL EXPLANATIONS AND THE GDPR}

Although the GDPR's 'right to explanation' is not legally binding, ${ }^{45}$ it has nonetheless connected discussion of data protection law to the longstanding question of how algorithmic decisions can be explained to

\footnotetext{
${ }^{42}$ For example, Kaiming He et al., Deep residual learning for image recognition, in PROCEEDINGS OF THE IEEE CONFERENCE ON COMPUTER VISION AND PATTERN RECOGNITION 770-778 (2016); Gao Huang et al., Deep networks with stochastic depth, in EUROPEAN CONFERENCE ON COMPUTER VISION 646-661 (2016).

${ }^{43}$ George A. Miller, The magical number seven, plus or minus two: some limits on our capacity for processing information., 63 PSYCHOL. REV. 81 (1956).

${ }^{44}$ Burrell, supra note 3.

${ }^{45}$ Wachter, Mittelstadt, and Floridi, supra note 1.
} 
experts as well as non-expert parties affected by the decision. Answering this question largely depends upon the intended purpose of the explanation; the information to be provided must be tailored in terms of structure, complexity and content with a particular aim in mind. Unfortunately, the GDPR does not define requirements for explanations of automated decision-making, and provides few hints as to the intended purpose of explanations of automated decision-making. Recital 71 of the GDPR, a non-binding provision, states that suitable safeguards against automated decision-making should be implemented and "should include specific information to the data subject and the right to obtain human intervention, to express his or her point of view, to obtain an explanation of the decision reached after such assessment and to challenge the decision."

This is the only time where an explanation is mentioned in the GDPR, leaving the reader with little insight into what type of explanation is intended, or what purpose it should serve. Based on the text, the only clear indication is that legislators wanted to clarify that some type of explanation can voluntarily be offered after a decision has been made. This can be seen as Recital 71 separates "specific information" which should be given before a decision is made, ${ }^{46}$ from safeguards that apply after a decision has been made 47 ("an explanation of the decision reached after such assessment [italics added]"). ${ }^{48}$ Further indications are not provided of the intended content of such ex post explanations.

The content of an explanation must reflect its intended purpose. Given the lack of guidance in the GDPR, many aims for explanations are feasible. Reflecting the GDPR's emphasis on protections and rights

\footnotetext{
${ }^{46}$ Jörg Hladjk, DS-GVO Art. 22 Automatisierte Entscheidungen im Einzelfall, in DATENSCHUTZ-GRUNDVERORDNUNG , Rn. 15 (Eugen Ehmann \& Martin Selmayr eds. 1 ed. 2017).

${ }^{47}$ The European Parliament makes the same distinction (information obligations vs. explanations of automated decisions) in their draft report on civil law rules on robotics when referring to the GDPR. See: EUROPEAN PARLIAMENT COMMITTEE ON LEGAL AFFAIRS, DRAFT REPORT WITH RECOMMENDATIONS TO THE COMMISSION ON CIVIL LAW RULES ON ROBOTICS (2016), http://www.europarl.europa.eu/sides/getDoc.do?pubRef=//EP//NONSGML\%2BCOMPARL\%2BPE582.443\%2B01\%2BDOC\%2BPDF\%2BV0//EN (last visited Nov 11, 2016); Sandra Wachter, Brent Mittelstadt \& Luciano Floridi, Transparent, explainable, and accountable AI for robotics, 2 SCI. RовOT. (2017).

${ }^{48}$ Hladjk, supra note 42 at Rn. 15-16 also supports this view that an explanation should be given after a decision has been taken, while recognising that this is not legally binding.
} 
for individuals, here we examine potential purposes for explanations from the perspective of the data subject. We propose three possible aims of explanations of automated decisions: to enhance understanding of the scope of automated decision-making and the reasons for a particular decision, to help contest a decision, and to alter future behaviour to potentially receive a preferred outcome. This is not an exhaustive list of potential aims of explanations, but rather reflects how the recipient of an automated decision, as with any type of decision, may wish to understand its scope, effects, and rationale, and take actions in response. In the following sections, we assess how these three purposes are reflected in the GDPR, and the extent to which counterfactual explanations meet and exceed the GDPR's requirements.

\section{A. EXPLANATIONS TO UNDERSTAND DECISIONS}

One potential purpose of explanations is to provide the data subject with understanding of the scope of automated decision-making, and the reasons that led to a particular decision. Several provisions in the GDPR can support a data subject's understanding of automated decisionmaking, although the types of information that must be shared tend to enhance broad understanding of automated decision-making systems, as opposed to the rationale of specific decisions. ${ }^{49}$ As a result, the GDPR does not appear to require opening the 'black box' to explain the internal logic of the decision-making system to data subjects. With this in mind, counterfactuals can provide information aligned with the GDPR's various informational requirements, while also providing some insight into the reasons that led to a particular decision. Counterfactuals, thus, could meet and exceed the requirements of the GDPR.

The description of explanations in Recital 71 does not include a requirement to open the 'black box'. ${ }^{50}$ Understanding the internal logic

\footnotetext{
${ }^{49}$ Wachter, Mittelstadt, and Floridi, supra note 1.

50 Id.; See also Article 29 Working Party's guidelines on automated individual decision-making ARTICle 29 DATA PROTECTION Working PARTY, Guidelines oN AUTOMATED INDIVIDUAL DECISION-MAKING AND PROFILING FOR THE PURPOSES OF REGULATION 2016/679 (2017), http://www.hldataprotection.com/files/2017/10/20171013_wp251_enpdf.pdf (last visited Oct 22, 2017)., which is very ambiguous but seems to support the claim that such a requirement is not only absent, but might not have been intended. On the one hand transparency in how decisions are made (Recital 71) appears to be very important (p. 16). However, at the same time, the guidelines state that the aim of Art 15(1)(h) is not to create individual explanations that require understanding the internal logic of the algorithm (p. 25). Hence, the guidelines suggest that Art 15(1)(h) calls for information about general system functionality, as is the case with its counterparts in Art 13(2)(f)
} 
of the algorithmic decision-making system is not explicitly required. Elsewhere, the GDPR contains transparency mechanisms (Art 12), notification duties (Art 13-14), and the right of access (Art 15), all of which create informational requirements concerning automated decision-making. Art 13-15 describe what kind of information needs to be provided if data are collected, either immediately when collected from the data subject (Art 13), the latest after a month when collected from a third party (Art 14), ${ }^{51}$ or at any time if requested from the data subject (Art 15) (see below). Among other things, Art 12 explains how this information (as defined in Art 13-14) should be conveyed. ${ }^{52}$ Art 1214 suggest that data subjects must be provided with "a meaningful overview of the intended processing" (Art12(7)), including "the existence of automated decision-making, including profiling, referred to in Article 22(1) and (4) and, at least in those cases, meaningful information about the logic involved, as well as the significance and the envisaged consequences of such processing for the data subject," (Art 13(2)(f), Art 14(2) $(\mathrm{g})$ ) as opposed to a detailed explanation of the internal logic of a system after a decision has been made. ${ }^{53}$ Rather they aim to offer a generic overview of intended processing activities, which enhances the data subject's understanding of the scope and purpose of automated decision-making. ${ }^{54}$

and Art 14(2)(g). This reading of Articles 13-15 would suggest that the Article 29 Working Party does not view non-binding Recital 71 as a requirement to explain the internal logic of individual decisions, as even the legally binding text in Article $15(1)(\mathrm{h})$, which is sufficiently vague to allow such an interpretation (see: Wachter, Mittelstadt, and Floridi, supra note 1, is not thought to create such a requirement. For further support that Recital 71 does not hinge on opening the black box, see: Martini, DS-GVO ART. 22 AUTOMATISIERTE ENTSCHEIDUNGEN IM EINZELFALL EINSCHLIEßLICH Profiling DAtenschutZ-GrundVERordnung Rn 35-37 (Paal \& Pauly eds., 1 ed. 2017).

${ }^{51}$ So called "just in time notification" ARTICLE 29 DATA PROTECTION WORKING PARTY, supra note 46 at 28.

${ }^{52}$ Dirk Heckmann \& Anne Paschke, DS-GVO Art. 12 Transparente Information, Kommunikation, in DATENSCHUTZ-GRUNDVERORDNUNG, Rn. 1-2 (Eugen Ehmann \& Martin Selmayr eds., 1 ed. 2017).

${ }^{53}$ Lorenz Franck, DS-GVO Art. 12 Transparente Information, Kommunikation, in DATENSCHUTZ-GRUNDVERORDNUNG VO (EU) 2016/679, Rn. 9-10 (Peter Gola ed., 1 ed. 2017); Sebastian Schulz, DS-GVO Art. 22 Automatisierte Entscheidungen im Einzelfall, in DATENSCHUTZ-GRUNDVERORDNUNG VO (EU) 2016/679 , Rn. 32-33 (Peter Gola ed., 1 ed. 2017); Suzanne Rodway, Just how fair will processing notices need to be under the GDPR, 16 PRIV. DATA PROT. 16-17 (2016).

${ }^{54}$ Christopher Kuner et al., Machine learning with personal data: is data protection law smart enough to meet the challenge?, 7 INT. DATA PRIV. LAW 1-2 (2017); Rosemary JAY, GUIDE TO THE GENERAL DATA PROTECTION REGULATION: A COMPANION TO DATA PROTECTION LAW AND PRACTICE 227 (4th Revised edition edition ed. 2017). 
Art 12(7) clarifies that the aim of Art 13-14 is to provide "in an easily visible, intelligible and clearly legible manner, a meaningful overview of the intended processing [italics added]." Two requirements are notable: (1) that the information provided must be meaningful to its recipient and broad in scope (a 'meaningful overview'), and (2) that the notification occurs prior to processing ('intended processing').

To understand what would constitute a 'meaningful overview', the envisioned medium of disclosure is instructive. Broadly applicable information appears to be required, rather than personalised disclosures. Legal scholars have suggested that notification duties can be satisfied via updates to existing privacy statements or notices ${ }^{55}$ (e.g. those displayed on websites or using QR codes) ${ }^{56}$ This requirement does not change based on the form of data collection. ${ }^{57}$ When data are collected from a third party (Art 14), an email sent to the data subject linking to the data controller's privacy statement(s) could suffice. ${ }^{58}$ The same holds true for personalised links ${ }^{59}$ referring to the privacy notice. Tools similar to those currently used to make users aware of the usage of cookies or monitoring shopping behaviour can be envisioned to satisfy the requirements in Art 14, thus making data subjects immediately aware of data collection. ${ }^{60}$ Detailed information appears to not be necessary as Art 12(7) states that the required information can be provided along with standardised icons. ${ }^{61}$ In trilogue, the European Parliament proposed several standardised icons that were ultimately not adopted (see Appendix 2). Despite this, the proposed icons reveal the initial expectations of regulators for simple, easily understood information. $^{62}$

\footnotetext{
${ }^{55}$ AlAin BENSOUSSAN, GENERAL DATA PROTECTION REGULATION: TEXTS, COMMENTARIES AND PRACTICAL GUIDELINES 113 (01 edition ed. 2017); Franck, supra note 49 at Rn. 12-13; JAY, supra note 50 at 223; Heckmann and Paschke, supra note 48 at Rn. 13-15; Rainer Knyrim, DS-GVO Art. 14 Informationspflicht bei Erhebung von Daten, in DATENSCHUTZ-GRUNDVERORDNUNG , Rn. 11-12 (Eugen Ehmann \& Martin Selmayr eds., 1 ed. 2017); ARTICle 29 Data ProteCtion Working PARTY, supra note 46 at 28.

${ }^{56}$ Lorenz Franck, DS-GVO Art. 13 Informationspflicht bei Erhebung von Daten, in DATENSCHUTZ-GRUNDVERORDNUNG VO (EU) 2016/679 , Rn. 34-35 (Peter Gola ed., 1 ed. 2017).

${ }^{57}$ ARTICLE 29 DATA PROTECTION WORKING PARTY, supra note 50 at 14.

${ }^{58}$ Knyrim, supra note 57 at Rn. 1-14.

${ }^{59}$ Franck, supra note 54 at Rn. 23-24.

${ }^{60}$ Knyrim, supra note 56 at Rn. 25-26.

${ }^{61}$ Id. at Rn. 9-10.; ARTICLE 29 DATA PROTECTION WORKING PARTY, supra note 51 at 28.

${ }^{62}$ The European Commission is tasked in Art 12(8) to develop such icons.
} 
These examples suggest Art 13-14 aim to provide a general overview of data processing that will be meaningful to all data subjects involved (e.g. all users of Twitter). The captive audience is more likely to be the general public or user base, not individual users, and their unique circumstances. ${ }^{63}$ This format of disclosure suggests notifications should be comprehensible to a general audience with mixed expertise and background knowledge. An "uneducated layperson" may be the envisioned audience for disclosures. ${ }^{64}$ This coincides with the general notion of Art 12(1) that all information and communication with the data subject has to be in a "concise, transparent, intelligible and easily accessible form," suggesting in-depth technical information and 'legalese' would be inappropriate. ${ }^{65}$ At a minimum, each provision suggests that information disclosures need to be tailored to their audience, with envisioned audiences including children (Recital 71) and uneducated laypeople.

Notifications regarding automated decision-making (Art 13(2)(f), Art 14(2)(g)) face particular constraints within an overall "meaningful overview." According to the, Article 29 Working Party, ${ }^{66}$ the UK Information Commissioner's Office ${ }^{67}$ and other commentators, ${ }^{68}$ informing the data subject about the "significance and envisaged consequences of automated decision-making" in a very simple manner, including "how profiling might affect the data subject generally, rather than information about a specific decision" will be sufficient. ${ }^{69}$ For instance, an explanation of how a low rating of creditworthiness can affect payment options, ${ }^{70}$ how intended data processing may result in a

\footnotetext{
${ }^{63}$ See Recital 58; Heckmann and Paschke, supra note 52 at Rn. 22. note that this information can also be provided orally. JAY, supra note 55 at 217. notes this too but warns that data controllers carry the burden to prove that the information was communicated.

${ }^{64}$ Franck, supra note 54 at Rn. 22. notes this for the elderly, uneducated people, foreigners or children; see also Heckmann and Paschke, supra note 52 at Rn. 17-18.

${ }^{65} \mathrm{JAY}$, supra note 56 at 218; Heckmann and Paschke, supra note 53 at Rn. 17-18; ARTICLE 29 DATA PROTECTION WORKING PARTY, supra note 51 at 13.

${ }^{66}$ ARTICLE 29 DATA PROTECTION WORKING PARTY, supra note 51.

${ }^{67}$ INFORMATION COMMISSIONER'S OFFICE, FEEDBACK REQUEST - PROFILING AND AUTOMATED DECISION-MAKING 15-16 (2017), https://ico.org.uk/media/about-theico/consultations/2013894/ico-feedback-request-profiling-and-automated-decisionmaking.pdf (last visited May 6, 2017).

${ }^{68}$ Rodway, supra note 54; Paal, DS-GVO Art. 13 Informationspflicht bei Erhebung von personenbezogenen Daten bei der betroffenen Person, in DATENSCHUTZ-

GRUNDVERORDNUNG (Paal \& Pauly eds., 1 ed. 2017).

${ }^{69}$ INFORMATION COMMISSIONER's OFFICE, supra note 68 at 16.

${ }^{70}$ Paal, supra note 69 at Rn. 31-32; INFORMATION COMMISSIONER's OFFICE, supra note 68 at 16.
} 
credit or job application being declined, ${ }^{71}$ or how driving behaviour might impact insurance premiums would be sufficient. ${ }^{72}$ Similarly, "meaningful information about the logic involved" is said to require only "clarification of the categories of data used to create a profile, the source of the data; and why this data is considered relevant" ${ }^{\text {"73 }}$ as opposed to a "detailed technical description about how an algorithm or machine learning works." 74

This view is echoed in the Article 29 Working Party's guidelines on automated individual decision-making, as they state that "details of the main characteristics considered in reaching the decision, the source of this information and the relevance" should be provided under Art 13$14 .^{75}$ Further, the "controller should find simple ways to tell the data subject about the rationale behind, or the criteria relied on in reaching the decision without necessarily always attempting a complex explanation of the algorithms used or disclosure of the full algorithm." It is however important to note that this requirement, despite referring to the decision-making 'rationale', seems to refer to general system functionality rather than an explanation of an individual decision. ${ }^{77}$ The guidelines state that Art 15(1)(h), which is seen to provide identical information as Articles 13(2)(f) and 14(2)(g), ${ }^{78}$ "implies a more general form of oversight, rather than a right to an explanation of a particular decision." 79 It is argued that the aim of these Articles is to demonstrate how automated process help data controllers to make more accurate, unbiased and responsible decisions and illustrate how the data,

\footnotetext{
${ }^{71}$ Rodway, supra note 54.

${ }^{72}$ ARTICLE 29 DATA PROTECTION WORKING PARTY, supra note 51 at 14-15.

${ }^{73}$ INFORMATION COMMISSIONER'S OFFICE, supra note 68 at 15.

74 Id. at 15-16. See also: Eugen Ehmann, DS-GVO Art. 15 Auskunftsrecht der betroffenen Person, in DATENSCHUTZ-GRUNDVERORDNUNG , Rn. 16-17 (Eugen Ehmann \& Martin Selmayr eds., 1 ed. 2017), who argues that Art 15 only entitles the data subject to know about the abstract logic and principles of data processing, but not the formula or code. Reference is made to Recital 63 in the English and French versions of the GDPR to support this claim. See also ARTICLE 29 DATA PROTECTION WORKING PARTY, supra note 51 at 14., which states "The controller should find simple ways to tell the data subject about the rationale behind, or the criteria relied on in reaching the decision without necessarily always attempting a complex explanation of the algorithms used or disclosure of the full algorithm."

${ }^{75}$ ARTICLE 29 DATA PROTECTION WORKING PARTY, supra note 51 at 14.

${ }^{76} \mathrm{Id}$. at 14.

${ }^{77}$ For an in-depth analysis between systems functionality and rationale of a decision see Wachter, Mittelstadt, and Floridi, supra note 1.

${ }^{78}$ ARTICLE 29 DATA PROTECTION WORKING PARTY, supra note 51 at 15.

${ }^{79}$ Id. at 24.
} 
characteristics, and method used are suitable to achieve this goal. ${ }^{80}$ In other words, the process of decision-making and the algorithms itself do not need to be fully disclosed, but rather a description of the logic of the algorithm, which may include a list of data sources or variables. ${ }^{81}$

Each disclosure under Art 13-14 must occur prior to data processing (Art 12(7)) or at the time of data collection, but before automated decision-making starts. ${ }^{82}$ Evidence of this is seen in the future-oriented language used in Art 13(2)(f) and Art 14(2)(g)), ${ }^{83}$ the obligation for information about the necessity of providing data for processing (Art 13(2)(e)), the clarification in Art 12(7) that information must be provided about "intended processing," and other provisions and jurisprudence. ${ }^{84}$ For automated decision-making, it is essential that

${ }^{80}$ Id. at 14.
${ }^{81}$ Paal, supra note 69 at Rn. $31-32$. sees no difference between Art $13-15$ in terms what
kind of information needs to be provided. See also: Paal, DS-GVO Art. 15
Auskunftsrecht der betroffenen Person, in DATENSCHUTZ-GRUNDVERORDNUNG, Rn.
31 (Paal \& Pauly eds., 1 ed. 2017). Further support is offered by the text of Recital 51
proposed by the European Parliament during Trilogue, which referred to "the general
logic of the data that are undergoing the processing and what might be the
consequences of such processing." See: EUROPEAN PARLIAMENT COMMITTEE ON CIVIL
LIBERTIES, JUSTICE AND HOME AFFAIRS, REPORT ON THE PROPOSAL FOR A REGULATION
OF THE EUROPEAN PARLIAMENT AND OF THE COUNCIL ON THE PROTECTION OF
INDIVIDUALS WITH REGARD TO THE PROCESSING OF PERSONAL DATA AND ON THE FREE
MOVEMENT OF SUCH DATA (GENERAL DATA PROTECTION REGULATION) - A7-
$0402 / 2013$
(2013), http://www.europarl.europa.eu/sides/getDoc.do?type=REPORT\&reference=A7-20130402\&language $=$ EN (last visited Nov 10, 2016).

${ }^{82}$ Wachter, Mittelstadt, and Floridi, supra note 1. Also, Franck, supra note 41; Knyrim, supra note 40; JAY, supra note 39 argue that the notification duties in Art 13 need to apply before the data is collected. Franck, supra note 57 at Rn. 33. links this to Art 13(2)(e) that obligates the data controllers to state whether the provision of personal data is a statutory or contractual requirement, or a requirement necessary to enter into a contract, as well as whether the data subject is obliged to provide the personal data and of the possible consequences of failure to provide such data'. Information about the necessity to provide data must therefore be given before the data is collected. See also ARTICLE 29 DATA PROTECTION WORKING PARTY, supra note 51 at 20 .

${ }^{83}$ Wachter, Mittelstadt, and Floridi, supra note 1.

${ }^{84}$ Rainer Knyrim, DS-GVO Art. 13 Informationspflicht bei Erhebung von Daten, in DATENSCHUTZ-GRUNDVERORDNUNG, Rn. 59-64 (Eugen Ehmann \& Martin Selmayr eds., 1 ed. 2017); Knyrim, supra note 55 at Rn. 13-14 notes that prior notification is also in line with the Bara and others judgment of the ECJ (C-201/14; 1.10.2015) which will have major implication for the GDPR as it shows that the court views prior notification of data transfer as essential. The ruling said that when information is gathered from a third party and transferred to another data controller for further processing (e.g. based on Member State law) prior notification of the data subject even if no consent is required - is essential. Not least because it enables the exercise of Art 15 (right of access) and Art 16 (right to data rectification) as soon as data is collected. 
information is provided before the start, else the right not to be subject of an automated decision can never be realised. The data subject has no chance to assess the associated risks, ${ }^{85}$ or whether one of the grounds in Art 22(2) (contract, law, explicit consent) actually apply that allow automated decision-making. If notifications do not occur prior to processing or decision-making, data subjects would only be able to contest decisions after the fact. This can be time and cost intensive, and unable to repair financial or reputational damage. Hence, one purpose of Art 13-14 is to make the data subject aware of future ${ }^{86}$ processing and to allow them to decide if they want their data to be processed (e.g. consent), ${ }^{87}$ assess the legitimacy (based on Member State law or contract) or exercise other rights in the GDPR. ${ }^{88}$

\section{Broader possibilities with the right of access}

The requirement for notification prior to processing applies only to the notification duties (Art 13-14). In contrast, the right of access (Art 15) can be invoked at any time by the data subject, opening up the possibility of providing information available after a decision has been made (i.e. the reasons for a specific decision). Von Lewinski ${ }^{89}$ argues that Art 15 (1)(h) grants a quasi-right to explanation of the main reasons connected to "meaningful information about the logic involved" which can be requested by the data subject after a decision has been made. Others argued that the information supplied via notification duties and the right of access is largely identical, meaning the right of access is similarly limited in terms of the scope of "meaningful information about the logic involved as well as the significance and the envisaged

\footnotetext{
${ }^{85}$ ARTICle 29 DATA Protection WORKING PARTY, supra note 51 at 13.

${ }^{86}$ Franck, supra note 54 at Rn. 45-49.

${ }^{87}$ On the importance to inform the data subject accurately (e.g. risks, safeguards, rights, consequences etc.) to allow for informed/explicit consent, see: JAY, supra note 55 at 218; Heckmann and Paschke, supra note 52 at Rn. 17-18; Schulz, supra note 54 at Rn. 32-33. ARtiCle 29 DATA PROTECTION WORKING PARTY, supra note 51 at 20. ${ }^{88}$ See also: Article 29 DAta Protection Working PARTy, Opinion 10/2004 ON $\begin{array}{lllll}\text { MORE HARMONISED INFORMATION PROVISIONS } & 29 & \text { (2004), }\end{array}$ http://ec.europa.eu/justice/policies/privacy/docs/wpdocs/2004/wp100_en.pdf (last visited Nov 10, 2016). on the need to provide meaningful information (to raise awareness) about data collection and the need to move away from long privacy statements. See also: Heckmann and Paschke, supra note 52 at Rn. 53-54.

89 von Lewinski, DS-GVO ARTIKEL 22 AUTOMATISIERTE ENTSCHEIDUNGEN IM EINZELFALL EINSCHLIEßLICH PROFILING BECK'SCHER ONLINE-KOMMENTAR DATENSCHUTZRECHT Rn. 54 (Wolff \& Brink eds., 21 ed. 2017) argues that Art 13(2)(f) and $14(2)(\mathrm{g})$ aim to inform about the outcome of a decision and that Art 15(1)(h) aims to inform about the main reasons, the logic and consequences of the decision.
} 
consequences" (Art 15(1)(h)). Information can thus largely be provided with identical tools (e.g. generic icons, privacy statements) ${ }^{90}$ or generic templates ${ }^{91}$ used for both notification and in response to access requests.

The narrower interpretation appears to be correct. ${ }^{92}$ The Article 29 Working Party supports this view, explaining that the information requirements in Art 13(2)(f), 14(2)(g), and 15(1)(h) are identical, ${ }^{93}$ while "Article 15 implies a more general form of oversight, rather than a right to an explanation of a particular decision." 94 A similar argument has been made by the ICO stating that Articles 13-15 aim to "provide information about how profiling might affect the data subject generally, rather than information about a specific decision." 95 The intention of Art 15 is to provide a control mechanism for data subjects to request at any time more or less the same information as Art 13-14, without having to rely on legal compliance with the notification duties by data controllers. $^{96}$ Many commentators also do not explicitly distinguish between types of information to be provided under Art 13-14 and Art 15 , suggesting that more detailed or personalised information is not required under the right of access. ${ }^{97}$ Reflecting this, much of Art 15 is identical in wording to Art 13-14. However, even with the overlap in wording, it must be noted that Art 12(7) - which states that a meaningful overview of the intended processing has to be provided at the latest at the time of the data collection - only refers to Art 13-14 and not Art 15. Further, Art 13-14 offer generic information for a broad audience (e.g. privacy statements on web pages), whereas requested information via Art 15 may need to be tailored according to the request of the user (e.g. to ask for confirmation of identity if in doubt (Art 12(6)). With that said,

\footnotetext{
${ }^{90}$ Paal, supra note 59 at Rn. 31; Franck, supra note 38 at Rn. 49; Lorenz Franck, DSGVO Art. 15 Auskunftsrecht der betroffenen Person, in DATENSCHUTZ-

GRUNDVERORDNUNG VO (EU) 2016/679 , Rn. 2-5 (Peter Gola ed., 1 ed. 2017);

Heckmann and Paschke, supra note 37 at Rn. 33-34.

${ }^{91}$ Franck, supra note 38 at Rn. 12-13 argues templates will be helpful, because as soon as Art 15 is lodged data controllers have to inform about all the information in Art 15, regardless of the actual request. See also: Ehmann, supra note 76 at Rn. 18.

${ }^{92}$ Wachter, Mittelstadt, and Floridi, supra note 1.

93 ARticle 29 DATA PROTECTION WORKING PARTY, supra note 51 at 15.

${ }^{94}$ Id. at 24.

${ }^{95}$ INFORMATION COMMISSIONER's OFFICE, supra note 68 at 16.

${ }^{96}$ Ehmann, supra note 76 at Rn. 4-5.

${ }^{97}$ Paal, supra note 84 at Rn. 31; JAY, supra note 55 at 228, 245; Rodway, supra note 54; Franck, supra note 93 at 16-17; BENSOUSSAN, supra note 56; Mario Martini \& David Nink, Wenn Maschinen entscheiden... - vollautomatisierte Verwaltungsverfahren und der Persönlichkeitsschutz, 36 NEUE Z. FÜR VERWALTUNGSRECHT (2017), https://dopus.unispeyer.de/frontdoor/index/index/docId/2442 (last visited Dec 18, 2017).
} 
the GDPR indicates a restricted scope for the right of access when compared to Art 13-14. Personal data of other data subjects must not be disclosed, as this could infringe their privacy. Access requests can also contravene trade secrets or intellectual property rights (Art 15(4) and Recital 63), meaning an appropriate balance between the data subject and controller's interests must be struck. ${ }^{98}$

\section{Understanding through counterfactuals}

Counterfactual explanations both meet and exceed the aims and requirements of the GDPR's transparency mechanisms (Art 12), notification duties (Art 13-14), and right of access (Art 15) which provide data subjects with understanding of the scope of automated decision-making. As argued above, Recital 71 does not give any clear indication of the intended purpose or content of explanations, including whether the internal logic of the algorithm must be explained. By providing simple 'if-then' statements, counterfactuals align with the requirement to communicate information to data subjects in a "concise, transparent, intelligible and easily accessible form" (Art 12(1)). They simultaneously provide greater insight into the data subject's personal situation and the reasons behind relevant automated decisions than an overview tailored to a general audience. Counterfactuals are also less likely to infringe on trade secrets or the rights and freedoms of others (e.g. privacy), since no data of other data subjects or detailed information about the algorithm needs to be disclosed, in line with restrictions on the right of access (Art 15(4) and Recital 63).

Perhaps most importantly, counterfactuals offer an explanation of some of the rationale of specific automated decisions, without needing to explain the internal logic of how a decision was reached (beyond a specific, limited set of dependencies between variables and the decision). This type of information is in line with the guidance mentioned above from the Article 29 Working Party ${ }^{99}$ and the UK's ICO. ${ }^{100}$ While opening the black box is not legally required, some information about the "logic involved" in automated decision-making must be provided (Articles 13(2)(f), 14(2)(g), 15(1)(h)). Under the Data Protection Directive's right of access, disclosing the algorithm's source code, formula, weights, full set of variables, and information about

\footnotetext{
${ }^{98}$ Franck, supra note 93 at Rn. 26-27; Ehmann, supra note 76 at Rn. 30-31.

${ }^{99}$ ARTICLE 29 DATA PROTECTION WORKING PARTY, supra note 51.

${ }^{100}$ INFORMATION COMMISSIONER's OFFICE, supra note 68.
} 
reference groups has generally not been required. ${ }^{101}$ The GDPR's right of access is likely to present similar requirements. Counterfactuals largely follow this precedent by disclosing only the influence of select external facts and variables on a specific decision. Although Art 13(2)(f), Art 14(2)(g), and Art 15(1)(h) do not require information about specific decisions (see section 'Explanations to Understand Decisions'), counterfactuals represents a minimal form of disclosure to inform the data subject about the "logic involved" in specific decisions. This form of disclosure regulatory burden for data controllers is minimised, as resolving the technical difficulties of interpretability or explaining the internal logic of complex systems to non-experts is not required to compute and communicate counterfactual explanations. Counterfactuals can thus be recommended as a minimally burdensome and disruptive technique to help data subject's understand the rationale of specific decisions beyond the explicit legal requirements of Art 13(2)(f), Art 14(2)(g), and Art 15(1)(h).

\section{B. EXPLANATIONS TO CONTEST DECISIONS}

Another possible purpose of explanations is to provide information that helps contest automated decisions when an adverse or otherwise undesired decision is received. A right to contest decisions is provided as a safeguard against automated decision-making in Art 22(3).

Contesting a decision can aim to reverse or nullify the decision and return to a status where no decision has been made, or to alter the result and receive an alternative decision. If the reasons that led to a decision have to be explained, the affected party can assess whether these reasons were legitimate and contest the assessment as required.

How a decision can be contested depends on whether the safeguards in Art 22(3) (i.e. rights to obtain human intervention, express views, and contest the decision) are interpreted as a unit that must be invoked together, or as individual rights that can be invoked separately or in any possible combination. ${ }^{102}$ To gauge the scope of explanations according to their purpose and aim, different possible models for contesting an automated decision need to be assessed.

Four models are possible. If the safeguards are a unit and must be invoked together, it is likely that some human involvement is

\footnotetext{
${ }^{101}$ For an in depth analysis of this jurisprudence, see: Wachter, Mittelstadt, and Floridi, supra note 1.

${ }^{102}$ Martini and Nink, supra note 100.
} 
necessary to issue a new decision. This could either be a human making the decision without any algorithmic help, hence the new result is a human decision rather than an automated decision. Alternatively, a person could be required to make a decision taking the algorithmic assessment and/or the data subject's objections into account, which would be human assessment with algorithmic elements. In both cases data subjects would lose their safeguards against the subsequent decision (Art 22 (3)), as both types of decision are not based "solely on automated processing" and thus do not meet the definition of automated individual decisions in Art 22(1). Another possibility is that a person could be required to monitor the input data and processing (e.g. based on the data subject's objections), with a new decision made solely by the algorithmic system. In this case the Art 22(3) safeguards still apply to the new decision. ${ }^{103}$

Finally, if the safeguards can be separated, and data subjects can invoke their right to contest the decision without invoking their right to obtain human intervention or express their views, a new decision could be issued with no human involvement. This decision could be contested again under Art 22(3). Which of these models will be preferred following implementation of the GDPR is unclear. ${ }^{104}$

The question remains what explanations are necessary or would be helpful to contest decisions. This will depend on the contesting model. The first model where a human makes a new decision and disregards everything the algorithm suggested, an explanation of the rationale of the original decision could be informative, but will not practically impact on the new decision made entirely by a human decision-maker. For each of the other models, where algorithmic

\footnotetext{
${ }^{103}$ However, if the new automated decision is communicated to the data subject by a person, it may not be considered "solely automated" and not subject to the Art 22(3) safeguards. The precise limitations on "solely automated" in Art 22(1) remain unclear. See also ARticle 29 DAtA Protection Working PARTY, supra note 35 at 10 which explains that fabricated human involvement should not be used as a loophole.

${ }^{104}$ Each interpretation is possible, as indicated by the European Parliament's proposal to add the following text to Article 20 in an earlier draft of the GDPR: "The suitable measures to safeguard the data subject's legitimate interests referred to in paragraph 2 shall include the right to obtain human assessment and an explanation of the decision reached after such assessment." This text clarified that a human would need to assess the decision in question. However, this text was not adopted in the end, which leaves implementation of any of the four models possible. With that said, treating the safeguards as individually enforceable may be the most sensible option. Individuals can have an interest in expressing their views or obtaining human intervention when a decision is poorly or misunderstood. Both interests do not, however, necessarily lead to challenging the decision, particularly if challenges are costly or have a low likelihood of success.
} 
involvement is envisioned, an explanation of the rationale of the decision could be helpful to identify potential grounds for contesting, such as inaccuracies in the input data, problematic inferences, or other flaws in the algorithmic reasoning. ${ }^{105}$

Even though an explanation of the rationale of a decision could be helpful, it does not imply an explanation is required by the GDPR, or is the intended aim of the non-binding right to explanation (Recital 71). Recital 71 does not specify the aim of the right or what information should be revealed, and does not explicitly require the algorithm's internal logic to be explained. An explicit link is not made with the right to explanation serving as a basis for a right to contest, ${ }^{106}$ not the least because the safeguards in Art 22(3) could be seen as independent from each other. Therefore, explanations under Recital 71 are not a necessary precondition to contest unfavourable decisions, even though this might be helpful.

Similarly, an explicit link has not been made between the right to contest and the transparency mechanisms (Art 12), notification duties (Art 13-14), right of access (Art 15), meaning the information provided through these rights and duties need not be explicitly tailored to help data subjects successfully contest decisions. ${ }^{107}$

Nonetheless, information provided by Art 12-15 may be helpful for contesting. Support is evident in the fact that notification duties aim to facilitate the exercise of other rights in the GDPR (Art 12(2)) to increase individual control over personal data processing. To achieve this, Articles 13(2)(b), 14(2)(c) and 15(1)(e) obligate data controllers to inform data subjects about their rights (Art 15-21) ${ }^{108}$ at the time when the data is collected (Art 13), within one month when obtained from a third party (Art 14), or at any time if requested by the data subject (Art

\footnotetext{
${ }^{105}$ Brent Mittelstadt et al., The ethics of algorithms: Mapping the debate, 3 BIG DATA SoC. (2016), http://bds.sagepub.com/lookup/doi/10.1177/2053951716679679 (last visited Dec 15, 2016).

${ }^{106}$ Hladjk, supra note 31 at Rn. 15-16; Schulz, supra note 38 at Rn. 33-37 argue that "contesting" and "explaining" the decision are separate and independent safeguards.

107 Similarly ARTICLE 29 DATA PROTECTION WORKING PARTY, supra note 35 argues that transparency in processing is essential to contesting, and that the reasons for the decisions and the legitimate basis should be known. However, the guidelines leave open whether this requires opening the black box and disclosing the algorithm. This seems unlikely as the guidelines state that not even Art 15(1)(h) aims to offer an explanation about an individual decision (p.24). Hence it can be assumed that the information provided does not need to include an explanation of the internal logic of a specific decision.

${ }^{108}$ If invoked via Art 15(1)(e), data controllers only have to inform about the rights enshrined in Art 16-21, excluding Art 20.
} 
15). However, Art 22 appears not to be covered by these provisions due to the odd phrasing of the obligation to inform of 'the existence of automated decision-making, including profiling, referred to in Article 22(1) and (4) and, at least in those cases, meaningful information about the logic involved, as well as the significance and the envisaged consequences of such processing for the data subject'.

As argued above, Art 13-15 will provide a meaningful overview of automated decision-making tailored to a general audience. On the surface, such an overview is not immediately useful for contesting decisions, as information about the rationale of individual decisions is not provided. In describing information to be provided about automated decision-making, these Articles explicitly refer only to Art 22(1) and (4). It follows that data subjects do not need to be informed about the safeguards against automated decision-making such as the right to contest (Art 22 (3)). ${ }^{109}$ This limitation is telling. If the aim of Art 13-15 were to facilitate contesting decisions by providing useful, individuallevel information, one would expect the right to contest or Art 22(3) to be explicitly discussed. Similarly, Art 13-15 seem not required to inform the data subject about their right not to be subject to an automated individual decision (Art 22(1)), from which a right to contest decisions could be inferred. ${ }^{110}$ In fact, in an earlier draft of the GDPR it was suggested that the information rights should refer to Art 20 as a

\footnotetext{
${ }^{109}$ Schulz, supra note 38 at Rn. 33-37 argues that data controllers only have to inform about the safeguards after an adverse decision has been issued. In fact, Art 12(3) introduces a very complicated model where the data controller has to inform upon request what kind of measures have been taken to satisfy a request under Art 15-22, without being informed about the safeguards beforehand. See also Martini, supra note 40 at $\mathrm{Rn} 39-40$, who acknowledges this loophole.

${ }^{110}$ It is important to note that Art 13-15 obligate to inform about the general right to object to processing (which forces data controllers to stop processing) in Art 21. Together with the information about the legitimate basis for processing provided in Art 13(1)(c) and Art 14(1)(c), this information could be used to contest decisions. However, this arrangement may place an unreasonable burden on the data subject. Contesting should be made as easy as possible, not least because the chances of successfully forcing the data controller to stop processing under Art 21 are different than under Art 22. For example, legitimate interest of data controller can trump a data subject right to object to data processing under Art 21. However, Art 22 does not allow automated decision-making on the basis of legitimate interest of the data controller (only explicit consent, law, or contract). This information will be useful for a data subject if they want to prevent data controllers from making decisions or to contest decisions.
} 
whole. ${ }^{111}$ Ultimately, this approach was not adopted, suggesting that the lack useful information for contesting decisions was intentional.

This lack of an explicit link to the safeguards against automated decision-making is in many ways unsurprising. Art 12-15 have the aim to inform data subjects about the existence of their rights in the GDPR, ${ }^{112}$ and to facilitate their exercise. ${ }^{113}$ This does not, however, mean that the controller is required to provide other information to help the data subject to exercise her rights. ${ }^{114}$ Rather, the data subject only needs to be informed about the existence of her rights, and provided with the necessary infrastructure for their exercise ${ }^{115}$ (e.g. web portals for complaints), including the elimination of unnecessary bureaucratic hurdles, ${ }^{116}$ a guarantee of reasonable response time to queries lodged ${ }^{117}$ (Art 12(3)), and the opportunity to interact with someone who has the power to change the decision. ${ }^{118}$ However, the data subject remains responsible to exercise her rights independently. ${ }^{119}$ As one commentator notes, Art 15 does not create a duty to legal consultancy; ${ }^{120}$ rather, it is sufficient that the data controllers inform about the existing rights in the GDPR. Unfortunately, Recital 60, which vaguely states that "any further information necessary to ensure fair and transparent processing taking into account the specific circumstances and context in which the personal data are processed" should be provided to the data subject, does not offer additional assistance to the data subject. This provision was intentionally moved to the non-binding Recitals during trilogue negotiations. ${ }^{121}$ Data controllers thus do not have a legal obligation to

\footnotetext{
${ }^{111}$ EUROPEAN DIGITAL RIGHTS, COMPARISON OF THE PARLIAMENT AND COUNCIL TEXT ON THE GENERAL DATA PROTECTION REGULATION 131 (2016), https://edri.org/files/EP_Council_Comparison.pdf (last visited Nov 20, 2016).

112 Knyrim, supra note 87 at Rn. 1-2.

${ }^{113}$ Heckmann and Paschke, supra note 52 at Rn. 4-5; Ehmann, supra note 76 at Rn. 12.

${ }^{114}$ Heckmann and Paschke, supra note 52 at Rn. 23-24. believe that easily understood information about the rights (Art 15-22) is sufficient to facilitate their exercise.

${ }^{115}$ BENSOUSSAN, supra note 56 at 114.

${ }^{116}$ Heckmann and Paschke, supra note 52 at Rn. 24-25.

${ }^{117}$ Franck, supra note 54 at Rn. 24-28.

${ }^{118}$ ARTICLE 29 DATA PROTECTION WORKING PARTY, supra note 51 at 15.

${ }^{119}$ Heckmann and Paschke, supra note 52 at Rn. 24-25.

${ }^{120}$ Franck, supra note 93 at Rn. 14.

${ }^{121}$ The European Commission, European Council, and European Parliament in Art 14(1)(h) proposed to create a legal duty for data controllers to provide any further information beyond those in the notification duties to ensure fair and transparent data processing. See: EUROPEAN COMMISSION, REGULATION OF THE EUROPEAN PARLIAMENT AND THE COUNCIL ON THE PROTECTION OF INDIVIDUALS WITH REGARD TO THE PROCESSING OF PERSONAL DATA AND ON THE FREE MOVEMENT OF SUCH DATA (GENERAL DATA PROTECTION REGULATION) (2012), http://ec.europa.eu/justice/data-
} 
provide information that will be particularly useful for the data subject to exercise her other rights.

One final comparable restriction is notable concerning Art 16, the right for the data subject to rectify inaccurate personal data. Data controllers are not required to specify which records most influenced a specific automated decision, which could be extremely helpful to a data subject attempting to identify inaccuracies as grounds to contest a decision. If large amounts of personal data are held, then the subject may have to check tens of thousands of items for inaccuracies.

\section{Contesting through counterfactuals}

Art 13-15 thus do little to facilitate a data subject's ability to challenge automated decisions. Information is not provided about the safeguards in Art 22(3) (e.g. the right to contest). It appears that data subjects do not need to be informed of their right not to be subject to an automated decision, which itself could imply a right to contest objectionable automated decisions. Similarly, Recital 71 has neither an explicit link to contesting decisions nor to understanding the black box. Even though an explanation could be helpful, they do not appear to be intended as a precondition for challenging decisions. If explanations were a precondition for contesting decisions, they would appear in the legally binding text. To offer greater protection to data subjects, these information gaps should be closed, meaning data controllers should inform about the right not to be subject to an automated decision and its safeguards. However, each of these seemingly intentional limitations on the information provided to data subjects suggest that information about the internal logic of an automated decision-making system (in compliance with "meaningful information about the logic involved as well as the significance and the envisaged consequences" in Articles 13(2)(4), 14(2)(g), and 15(1)(h)), which could facilitate contesting decisions, does not need to be provided.

Given these restrictions, counterfactuals could be helpful for contesting decisions, and thus provide greater protection for the data subject than currently envisioned by the GDPR. Regardless of the legal status of the right to explanation, the right to contest is a legally binding

protection/document/review2012/com_2012_11_en.pdf (last visited Nov 10, 2016); EUROPEAN DigITAL RIGHTS, supra note 114 at 126-127, 129. However, this proposal was not adopted and moved to Recital 60, suggesting that there is no legal duty to provide more information than required in Art 13-14; see Franck, supra note 57 at Rn. 27-28. 
safeguard (Art 22(3)). By providing information about the external factors and key variables that contributed to a specific decision, counterfactuals can provide valuable information for data subjects to exercise their right to contest. This would also be in line with the guidelines of the Article 29 Working Party, which urge that understanding decisions and knowing their legal basis is essential for contesting decisions, and is not necessarily linked to opening the black box. ${ }^{122}$ An explanation that low-income led to a loan application being declined could, for example, help the data subject contest the outcome on the grounds of inaccurate or incomplete data regarding her financial situation. Understanding the internal logic of the system that led to income being considered a relevant variable in the decision, which would require a technical explanation unlike a counterfactual explanation (see Appendix 1), may be desirable in its own right, but is not absolutely necessary to contest the decision based on that variable.

Counterfactuals offer a solution and support for contesting decisions by providing data subjects with information about the reasons for a decision, without the need to open the black box. Although Art. 16 of the GDPR gives the data subject the right to correct inaccurate data used to make a decision, the data subject does not need to be informed which data the decision depended. Where a large corpus of data has been collected, an individual without knowledge of which data is relevant or most influential on a particular decision is forced to vet all of it. This lack of information increases the burden on data subjects seeking a different outcome. Counterfactuals provide a compact and easy way to convey these dependencies (i.e. which data was influential), and to facilitate effective claims that a decision was made on the basis of inaccurate data and contest it.

\section{EXPLANATIONS TO ALTER FUTURE DECISIONS}

From the view of the data subject, alongside understanding and contesting decisions, explanations can also be useful to indicate what could be changed to receive a desired result in the future. This purpose does not necessarily relate to the right to contest. Accurate decisions can produce unfavourable results for the data subject. The chances of successfully challenging the decision will also be low in some cases, or the costs and effort required too high. In these situations, the data subject may prefer to change aspects of her situation by adapting her

\footnotetext{
${ }^{122}$ ARTICle 29 DAta ProteCtion Working PARTY, supra note 51 at 16, 24.
} 
behaviour, and requesting a new decision once more favourable conditions exist.

Using explanations as a guide to altering behaviour to receive a desired automated decision is not directly addressed in the GDPR. This does not, however, undermine the interest data subjects have in receiving desired results from automated decision-making systems. For example, if a subject was rejected for a loan due to insufficient income, a counterfactual explanation will indicate if reapplying in the event of an immediate pay rise is reasonable. The Article 29 Working Party seems to agree, stating in relevant guidelines that "tips on how to improve these habits and consequently how to lower insurance premiums" could be useful for the data subject. ${ }^{123}$ For reasons outlined in Appendix 1, technical explanations that try to provide "meaningful information about the logic involved, as well as the significance and the envisaged consequences [italics added]" of automated decision-making are not guaranteed to be useful in this situation.

Counterfactuals can thus be useful for altering future decisions in favour of the data subject. By providing information about key variables and "close possible worlds" which result in a different decision, data subjects can understand which factors could be changed to receive the desired result. For decision-making models and environments with low variability over time, or models that are 'artificially frozen' in time for individuals (i.e. future decisions will be made with the same model as the individual's original decision), this information can help the data subject to alter her behaviour or situation to receive their desired result in the future.

With that said, unanticipated dependencies between intentionally changed attributes and other variables, such as an increase in income resulting from a change in career, may undermine the utility of counterfactuals as guides for future behaviour. Counterfactual explanations can, however, address the impact of changes to more than one variable on a model's output at the same time. Further, regardless of the utility of counterfactuals as guidance for future behaviour, their ability to help individuals understand which data and variables were influential in specific prior decisions remains unaffected.

${ }^{123} I d$. at 15 . 


\section{CONCLUSION}

We have proposed a novel lightweight form of explanation that we refer to as counterfactual explanations. Unlike existing approaches that try to provide insight into the internal logic of black-box algorithms, counterfactual explanations do not attempt to clarify how decisions are made internally. Instead, they provide insight into which external facts could be different in order to arrive at a desired outcome. ${ }^{124}$ Importantly, counterfactual explanations are efficiently computable for many standard classifiers, particularly neural networks. As our new form of explanation significantly differs from existing works, we have justified its nature as an explanation with reference to previous works in the philosophical literature and early A.I.

From the view of the data subject, we have assessed three purposes of explanations of automated decisions: understanding, contesting and altering. We compared these aims with the provisions of the GDPR and evaluated if they rely upon opening the black box. We concluded that the framework offers little support to achieve these goals, and does not mandate that algorithms are explainable to understand, contest or alter decisions.

The GDPR itself provides little insight into the intended purpose and content of explanations. Recital 71, the only provision that explicitly mentions explanations, does not reveal their intended purpose or content. Given the final text of the GDPR, it appears that explanations can voluntary be offered after decisions have been made, and are not a required precondition to contest decisions, Further, there is no clear link that suggests that explanations under Recital 71 require opening the black box.

Recognising this relative lack of insight into explanations, related provisions addressing automated decision-making were examined. Notification duties defined in Art 13-14 apply prior to data processing or before a decision is made (i.e. at the time of data collection), and provide a simple and generic overview of intended data processing activities that aims to inform a general audience (Art 12 (7)). This type of "meaningful overview" of automated decision-making is largely unsuitable to understand the rationale of specific decisions. Art

\footnotetext{
${ }^{124}$ The method described here is compatible with a proposal made by Citron and Pasquale to allow consumers to manually enter "hypothetical alterations" to their credit histories and view their effects. See: DANIELlE KEATS CitRon \& FRANK A. PASQUALE, THE SCORED SOCIETY: DUE PROCESS FOR AUtOMATED PREDICTIONS (2014), https://papers.ssrn.com/abstract=2376209 (last visited Mar 4, 2017).
} 
13-14 similarly do not facilitate contesting decisions, owing to a lack of information to be provided about the right not to be subject to automated individual decision-making (Art 22(1)), and its safeguards (Art 22(3)). The right of access (Art 15) provides nearly identical information to Art 13-14, and is thus offers similarly limited value for understanding and contesting decisions. The rights and freedoms of others (e.g. privacy or trade secrets) which are protected in Art 15(4) and Recital 63 pose an additional barrier to transparency when access requests are lodged. Across each of these Articles, technical explanations of the internal logic of automated decision-making systems are not legally mandated. Finally, offering explanations to give guidance how to receive the desired result in the future does not appear to be an aim of the GDPR, but could still be highly useful for individuals seeking alternative, more desirable outcomes.

Any future attempt to implement a legally binding right to explanation as a safeguard against automated decision-making within the framework provided by the GDPR faces several notable challenges. Automated decision-making must be based "solely on automated processing," and have "legal effects" or similarly significant effects (Art 22(1)). Additionally, exemptions from the safeguards against automated decision-making can be introduced through Member State law (Art 23). ${ }^{125}$

However, the data subject's desire to understand, contest, and alter decisions does not change based on these definitional issues. We therefore propose to move past the limitation of the GDPR and to use counterfactuals as unconditional explanations. These unconditional explanations should be given whenever requested, regardless of outcome (positive or negative decision), whether the decision was based on solely automated processes and their (legal or similar significant) effects.

Counterfactual explanations could be implemented in several ways. The transience of decision-making models suggests that counterfactuals either need to be computed automatically at the time a decision is made, or a copy of the model archived to compute counterfactuals at a later time. As multiple outcomes based on changes to multiple variables may be possible, a diverse set of counterfactual explanations should be provided, corresponding to different choices of nearby possible worlds for which the counterfactual holds. These sets

${ }^{125}$ These exemptions may be based for example on national security, the enforcement of civil law claims, or the protection of the data subject or the rights and freedoms of others. 
could be disclosed when automated decision-making occurs, or in response to specific requests lodged by individuals or a trusted third party auditor. ${ }^{126}$ In any case, disclosures should occur in a reasonable window of time (e.g. Art 12(3)).

Future research should determine appropriate distance metrics and requirements for a sufficient and relevant set of counterfactuals across use sectors and cases which have very different needs. While prior philosophical debate may prove helpful, the absence of causal models in most modern classifiers, as well as the preferences of the recipient(s) of the set, must be accounted for in choosing appropriate metrics and requirements. Compared to prior discussion of measuring "closest possible worlds," setting requirements for appropriate "close possible worlds" represents a very different philosophical, social, and legal challenge.

To minimise bureaucratic burdens for data controllers and delays for data subjects and third party auditors, automated calculation and disclosure of counterfactuals would be preferable. We recommend this type of automated implementation going forward. One possible approach is to provide individuals or third party auditors with access to 'auditing APIs', ${ }^{127}$ which allow users to request counterfactual explanations from the service provider, and perhaps compute them directly via the API. Access to (historical) decision-making models used for the decision at hand, as well as permissive terms of service that allow for such auditing, would be required. ${ }^{128}$ This functionality could potentially be embedded in existing APIs.

Counterfactual explanations provide reasons why a particular decision was received (e.g. low income), offer grounds to contest it (e.g. if the data controller used inaccurate data about the income of the

\footnotetext{
${ }^{126}$ Wachter, Mittelstadt, and Floridi, supra note 1.

${ }^{127}$ Who would shoulder the costs of hosting these APIs and computing counterfactuals is an important political issue that would require resolution. This issue goes beyond the scope of this paper. For related discussion of implementing algorithmic auditing, see: Christian Sandvig et al., Auditing algorithms: Research methods for detecting discrimination on internet platforms, DATA DISCRIM. CONVERT. CRIT. CONCERNS PRODUCT. INQ. (2014), http://social.cs.uiuc.edu/papers/pdfs/ICA2014-Sandvig.pdf (last visited Feb 13, 2016); Brent Mittelstadt, Auditing for Transparency in Content Personalization Systems, 10 INT. J. COMMUN. 12 (2016).

${ }^{128}$ Counterfactuals must be computed on the basis of the decision-making model at the time the decision was taken. Assuming automated decision-making models change over time, in implementations not involving automatic computation of counterfactuals at the time a decision is made (which may be cost prohibitive), it will be necessary for data controllers to keep 'audit logs' indicating the state of the decision-making model at the time of the decision.
} 
applicant), and provide limited 'advice' on how to receive the desired results in the future (e.g. an increase of 4000 pounds/ year would have resulted in a positive application). Their usage would help to resolve two primary objections to a legally binding right to explanation: first, that explaining the internal logic of automated systems to experts and nonexperts alike is a highly difficult and perhaps intractable challenge; and second, that an excessive disclosure of information about the internal logic of a system could infringe on the rights of others, either by revealing protected trade secrets or by violating the privacy of individuals whose data is contained in the training dataset. In contrast, counterfactuals allow an individual to receive explanations without conveying the internal logic of the algorithmic black box (beyond a limited set of dependencies), and are less likely to infringe the rights and freedoms of others than full disclosure. Assuming reasonable limitations are set on the number of counterfactuals that must be provided, counterfactuals are also less likely to provide information that reveals trade secrets or allows gaming of decision-making systems.

As a minimal form of explanation, counterfactuals are not appropriate in all scenarios. In particular, where it is important to understand system functionality, or the rationale of an automated decision, counterfactuals may be insufficient in themselves. Further, counterfactuals do not provide the statistical evidence needed to assess algorithms for fairness or racial bias. Given these limitations, more general forms of explanations and interpretability should still be pursued to increase accountability and better validate the fairness and functionality of systems.

However, counterfactuals represent an easy first step that balances transparency, explainability, and accountability with other interests such as minimising the regulatory burden on business interest or preserving the privacy of others, while potentially increasing public acceptance of automatic decisions. Rather than waiting years for jurisprudence to dissolve all these uncertainties, we propose to abandon the narrow definitions and conditions the GDPR imposes on automated decision-making, and offer counterfactuals as unconditional explanations at the request of affected individuals. 


\section{APPENDIX 1: SIMPLE LOCAL MODELS AS EXPLANATIONS}

As discussed in section 'Explanations in A.I. and Machine Learning', approaches such as LIME $^{129}$ that generating simple models as local approximations of decisions make a three-way trade-off between the quality of the approximation vs. the ease of understanding the function and the size of the domain for which the approximation is valid. ${ }^{130}$

To illustrate the instabilities of the approach with respect to the size of the domain, we consider a simple function of one variable. Even for problems such as this the notion of scale, or how large a region should an explanation try to describe is challenging, with the ideal choice of scale depending on what the explanation would be used for.

As a real-world example, consider being stopped by someone in a car who asks which direction they should travel in to go north. Fundamentally, this is a difficult question to answer well, with the most appropriate answer depending upon how far north they wish to travel. If they do not intend to travel far, simply pointing north gives them enough information, however, if they intend to travel further, roads that initially point north may double back on themselves or be cul-de-sacs and better directions are needed. If they intend to travel a long way, they may be better off ignoring the compass bearing entirely, and instead try to directly join up with an inter-city network.

This exact issue is faced when automating explanations of decisions: the generated explanations are generic, and designed to be useful to the recipient of the explanation regardless of how they are used. However, as shown in figure 1, the explanation -- or simplified model-- can vary wildly with the scale or range of inputs considered.

\footnotetext{
${ }^{129}$ Ribeiro, Singh, and Guestrin, supra note 22.

${ }^{130}$ Bastani, Kim, and Bastani, supra note 23; Lakkaraju et al., supra note 23.
} 

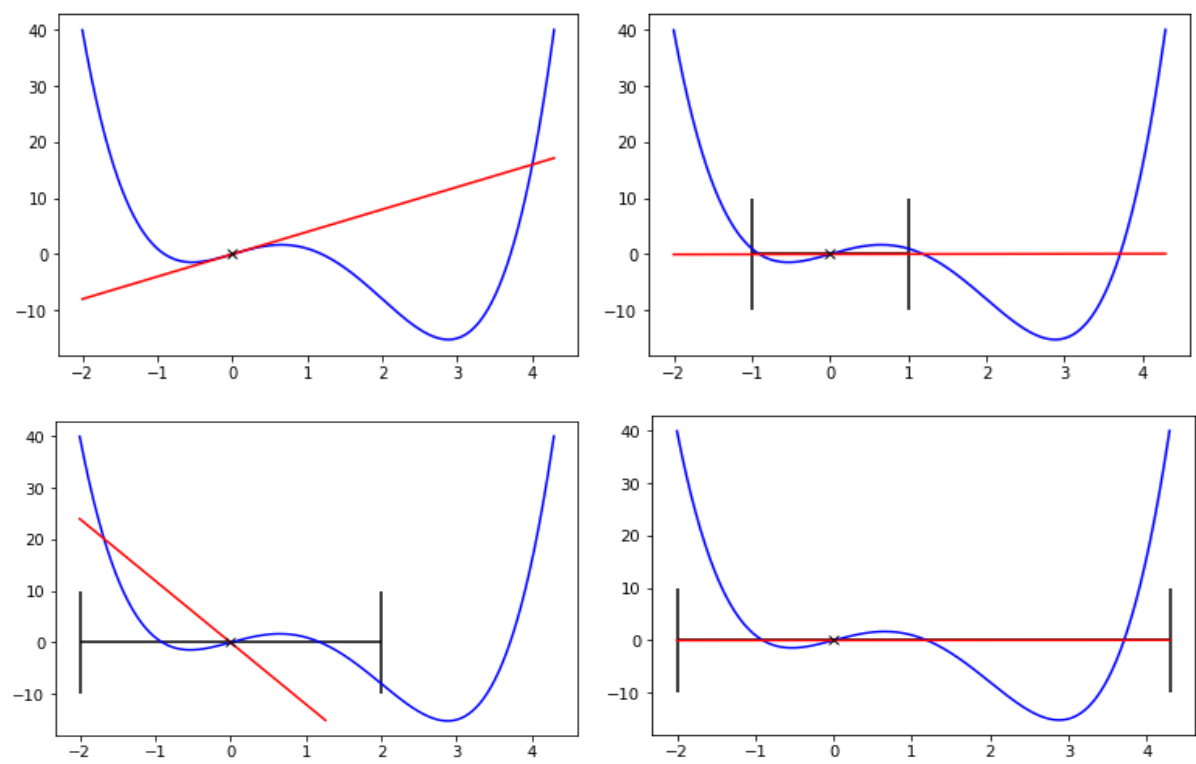

Figure 1 - Local models varying with choice of scale. The red line in each subfigure shows a local approximation of the same blue score curve centred at the same location in each plot. The varying range over which the approximation is computed is given by the region marked by black bars. Different choices of range e.g. top left vs. bottom left can lead to completely opposing explanations where the score either increases or decreases as the value along the bottom axis increase.

As can be seen, the direction and magnitude of the linear approximation (red) to underlying function (blue) vary dramatically with choice of domain, and deciding which approximation is most helpful to a layperson trying to understand the decision made about them is nontrivial.

To show the difficulties that would exist in either trying to use local models to either compute counterfactuals, or simply for the data subject to adjust their score, we assume that the subject desired to know how to obtain a lower score of -10 or below. In this case, none of the local approximations would be useful. The top left model, which is based on exact description of the function around point $\mathrm{x}$ predicts that a score of -10 would be obtained with a value of -2.5 -- corresponding to an actual score of 91.5, while the two centre approximations suggest that it is not possible to obtain any score except 0 , and the bottom left approximation says that -10 occurs at near 0.9 - which actually corresponds to a local maxima.

In contrast, the counterfactual explanation for a query such as "Why was the score not below -10?" would return the answer "Because the $\mathrm{x}$ value was not 2.15 " (the counterfactual is illustrated in figure 2 by 
the green dot). Of course, it should be noted that the two approaches are generally incomparable. In much the same way, if a data subject desired to know a local linear approximation about their data point, knowledge of counterfactuals would not be helpful. However, of the two approaches, counterfactuals are the only one that will provide some indication if it is worth reapplying for a loan in the event of a pay rise.

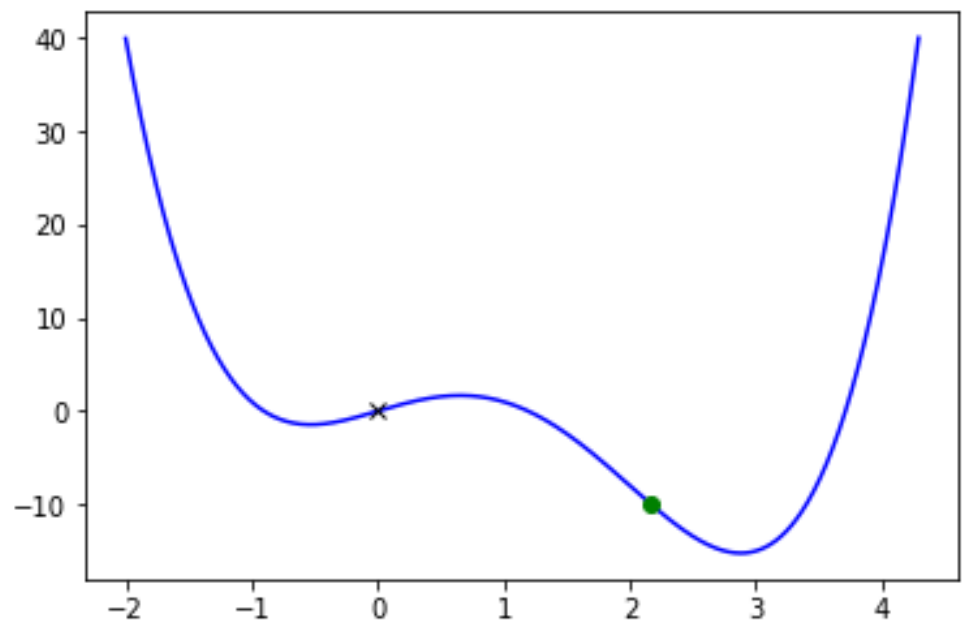

Figure 2 - Visual representation of the range of a counterfactual explanation 


\section{APPENDIX 2: EXAMPLE TRANSPARENCY INFOGRAPHIC}

The figure below shows several icons proposed by the European Parliament during trilogue that were ultimately not adopted as a standard. It gives an example of the level of complexity expected in an explanation to a data subject.

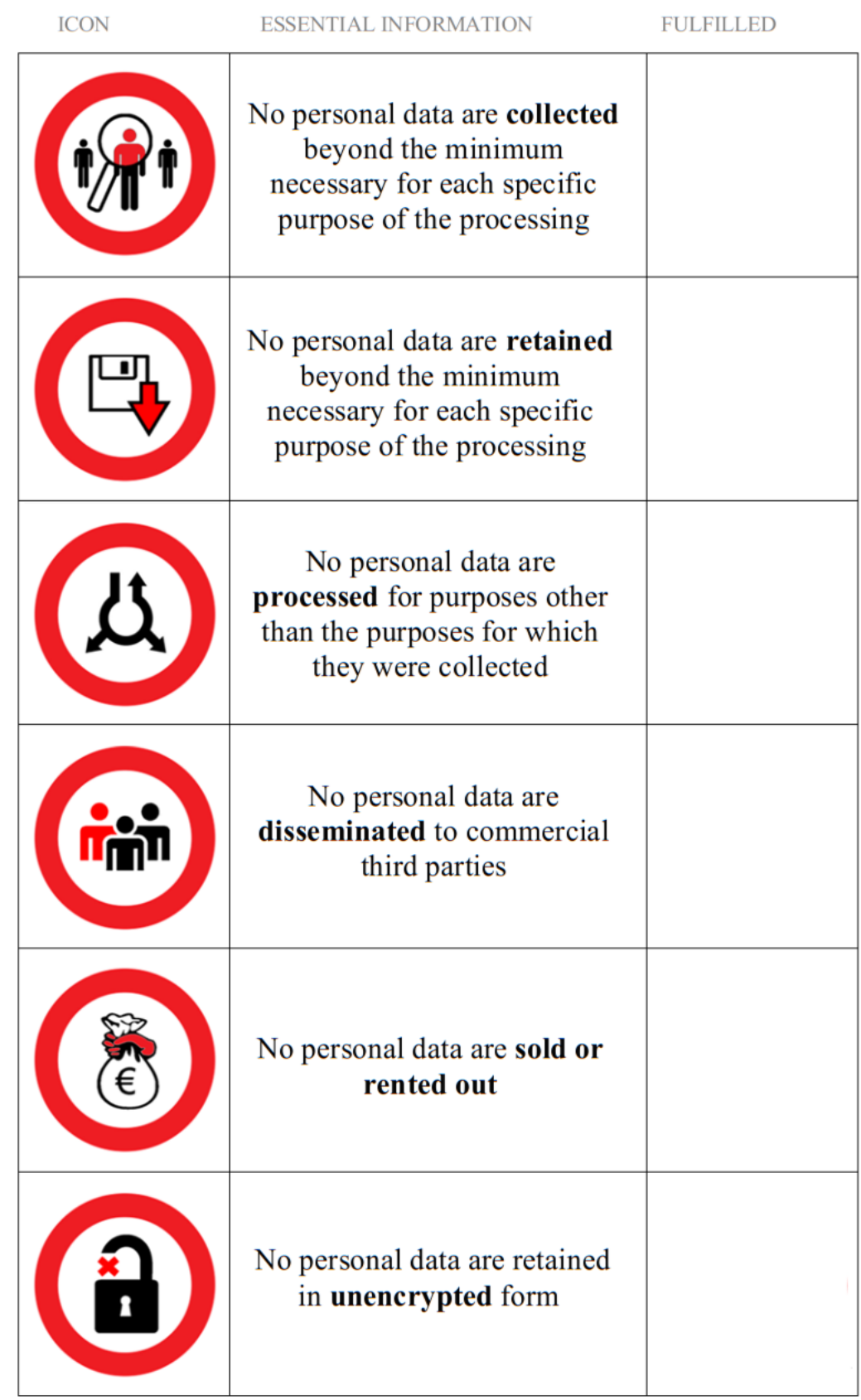

